\title{
A Dynamic Model of Unemployment with Migration and Delayed Policy Intervention
}

\author{
Liliana Harding1 • Mihaela Neamţu ${ }^{2}$
}

Accepted: 8 August 2016 / Published online: 17 August 2016

(C) The Author(s) 2016. This article is published with open access at Springerlink.com

\begin{abstract}
The purpose of this paper is to build and analyse a model of unemployment, where jobs search is open to both natives and migrant workers. Markets and government intervention respond jointly to unemployment when creating new jobs. Full employment of resources is the focal point of policy action, stimulating vacancy creation. We acknowledge that policy is implemented with delays, and capture labour market outcomes by building a non-linear dynamic system. We observe jobs separation and matching, and extend our model to an open economy with migration and delayed policy intervention meant to reduce unemployment. We analyse the stability behaviour of the resulting equilibrium for our dynamic system, including models with Dirac and weak kernels. We simulate our model with alternative scenarios, where policy action towards jobs creation considers both migration and unemployment, or just unemployment.
\end{abstract}

Keywords Unemployment · Dynamic models · Distributed delay · Hopf bifurcation · Migration · Matching

\section{Introduction}

Creating new job opportunities is a priority for any vibrant economy. In the aftermath of the financial crisis government efforts have primarily focused on containing insta-

Liliana Harding

liliana.harding@uea.ac.uk

Mihaela Neamţu

mihaela.neamtu@e-uvt.ro

1 School of Economics, University of East Anglia, Norwich Research Park, Norwich NR4 7TJ, UK

2 West University of Timişoara, Str. J. H. Pestalozzi, nr. 16, 300115 Timişoara, Romania 
bility and reducing public debt, yet active labour market policies might be needed to promote employment. New vacancies reduce the number of those registered as unemployed, but they conceivably attract as well new migrant workers. International migration can add to the uncertainty in labour market prospects for natives, and easily translates into a sensitive issue for policy makers to review in times of economic instability (Facchini and Mayda 2008; Blanchflower and Shadforth 2009; Hatton 2014). Arguably, a successful policy addressing unemployment and that benefits the economy over time creates employment opportunities for natives, as well as supporting migrants. We hence propose to explore the viability of a labour market policy supporting vacancy creation, where the government acknowledges the simultaneous search for jobs by natives and new immigrants.

We consider unemployment in migrants destinations as a factor in the latters' decision to move. Unemployment levels influence the potential to match migrants with employment opportunities abroad and it can act as a deterrent to further immigration. The empirical literature on labour mobility finds a weak and ambiguous relationship between unemployment and migration (see for a survey of relevant studies Bauer and Zimmermann 1999; Jean and Jimenez 2011; Angrist and Kugler 2003; Blanchflower and Shadforth 2009; Damette and Fromentin 2013). Yet, Hatton (2014) concludes that during an economic slump the increase in unemployment is typically associated with a decline in immigration. As such, we will accept that migration is declining where unemployment is on the rise, further reflecting the experience during the recent economic crisis.

The complex interaction between migration and unemployment over time motivated a dynamic model capturing developments in aggregate unemployment for an open economy. We build on insights from job search and matching models of unemployment [e.g. Pissarides (2011)] but focus on aggregate flows to and out of unemployment, along with migration and its labour market impact in an open economy. We analyse the fluctuation in unemployment in a continuous time framework, as previously proposed by Shimer (2011) when undertaking empirical tests of US unemployment factors. We explore more closely the dynamic market interaction between unemployment, vacancies, employment and migration, by adding policy intervention to the framework.

Earlier research considering the economic dynamics of migration is rare, but Camacho (2013) models the decisions of inter-regionally mobile skilled workers and analyses the steady state stability of a system informed by the new economic geography framework. The paper provided numerical simulations presenting stability trajectories in the context of different technologies. While also building a dynamic system and undertaking simulation for an open economy, we depart in this paper from the individual choices of migrants and focus instead on the aggregate outcomes in terms of unemployment, with migration a contributing factor.

Our approach is to build a system modelling the dynamics of unemployment and to question the extent to which the policy framework leads to locally stable outcomes. The local stability of our system is analysed theoretically under particular conditions, on the basis of a non-linear dynamic mathematical model with distributed delays. Earlier economic literature modelling delays in a different decision making context observes that lack of lags in information, while conventionally a source of stability, it can also destroy a stable equilibrium (Huang 2008). To test the impact of delays in 
policy reaction we simulate our system under alternative specifications. We observe a variation in outcomes where migration is built into the policy reaction function and enhances labour market intervention beyond the usual response to local unemployment.

Intervention in reaction to particular events has been previously considered by Nikolopoulos and Tzanetis (2003) who developed a model that looks at housing allocation of homeless families due to a natural disaster. Using concepts from this paper, Misra and Singh (2011) constructed and analysed nonlinear mathematical models for the reduction of unemployment. In Misra and Singh (2013), the model is further described by a nonlinear dynamic system with delay. Their system includes: the number of unemployed, the number of employed, and the number of newly created vacancies through government intervention. There are separations from existing jobs and posts are being occupied by a proportion of those unemployed, who also benefit from policy induced vacancies creation. A time delay is introduced in the rate of creation of new vacancies through policy action and a detailed stability analysis is provided.

Our study is primarily in line with that of Misra and Singh $(2011,2013)$. The latter started from a macroeconomic perspective in a developing country and observed aggregate matching processes and the interaction between unemployment and policy supported vacancies. We propose to extend the application of this framework to the case where migrants arrive to look for jobs, and can become unemployed or take up employment at destination. Our analysis reflects the circumstances in developed economies that are open to at least some internationally mobile workers. Under conditions similar to the single market operating in the EU there is no direct policy control over migrant numbers and governments just observe the stock of migrants on their territory along with the number of the unemployed. Consequently, any vacancies created through policy intervention will depend on the number of the unemployed observed simultaneously with new migrant labour.

In this paper we consider initially a new state variable to supplement the system described in Misra and Singh (2013), the total number of jobs on the market. This variable captures market responses to unemployment and the implicit downward pressures on wages. Due to the fact that "continuously distributed" delay models are more realistic (Ruan 1996), we also propose to use a distributed time delay in relation to both unemployment and migration. We further focus on the number of immigrants as a variable along with market and policy induced job creation. For the resulting nonlinear mathematical model we use stability theory of differential equations. We prove analytically that under some conditions there is a unique positive equilibrium point. Then, for this equilibrium point, we study the local stability behaviour and we analyse the influence of the distributed time delay on the stability properties. In this way, two different kernels are introduced and a detailed analysis is done with respect to these. For both Dirac and weak kernels we prove that Hopf bifurcation occurs and a family of periodic solutions bifurcates from the equilibrium when the bifurcation parameter passes through a critical value. This critical value of delay is obtained analytically.

The paper is organised as follows. In Sect. 2 the model for unemployment reduction with migration and two kernels is described. An equilibrium analysis is presented in 
Sect. 3. For different types of kernels a stability analysis is done in Sect. 4. Numerical simulations are carried out in Sect. 5. Finally, concluding remarks are given in Sect. 6 and the Appendix provides technical details.

\section{The Mathematical Model}

We model unemployment in a continuous time framework, along with a policy of jobs creation. The number of individuals claiming unemployment rises over time under the influence of external factors and we see this number diminish where a proportion of the unemployed find jobs created by recovering markets or through active labour market stimulation. Yet, some of those currently in employment might also be dismissed and job losses increase unemployment, along with the arrival of migrants who cannot find a job at destination. Finally, some of the unemployed can also leave or retire, diminishing the numbers of those in need of support through job creation. The change in unemployment is captured in Eq. (1).

In the modelling process, we consider that all individuals are qualified to do any job. At any time $t$, the number of unemployed persons, $U(t)$, changes by an autonomous factor $a_{1}$. The instantaneous rate of movement from unemployment to employment is jointly proportional to $U(t)$ and the number of available vacancies $P(t)+V(t)-E(t)$, where $P(t)$ is the total number of jobs on the market, $V(t)$ is the total number of newly created vacancies through government intervention and $E(t)$ is the number of employed individuals.

Migrants are attracted to a particular labour market by a mixture of economic and non-economic factors. Persistently large income differentials between migrants' typical origins and destination countries mean an ongoing inflow of workers from abroad, ready to enter the labour market of our observed economy. The stock of migrants typically increases, often through the attractiveness of social networks and translating into an autonomous rise of migrant stocks, independent of economic conditions. Yet migrants' attraction to a labour market is a function of their employability, which depends on the available jobs in the destination economy. Migrants can also benefit from access to newly created vacancies. By entering employment abroad, immigrants become part of the labour force at destination, rising the number of total employed individuals in the economy. Foreign workers can also be expected to register as jobless, ultimately increasing the numbers claiming unemployment benefits. On the other hand, return migration often represents a significant proportion of the initial migrants, diminishing the migrant stock along with natural attrition. Such developments are captured by Eq. (2).

In the model, the number of immigrants that become part of the active labour force at destination at time $\mathrm{t}$ is denoted by $M(t)$. It is assumed that migration increases by an autonomous amount $m_{1}$. The rate of instantaneous movement of immigrants into employment $m_{2}$ is jointly proportional to $M(t)$ and the number of available jobs $P(t)+V(t)-E(t)$. The proportion of migrants who register as unemployed is $a_{5}$.

The number of individuals in employment rises through the job findings of the unemployed and migrant workers occupying market created jobs and vacancies supported through government intervention. Total employment decreases as a con- 
sequence of either retirement or natural loss, along with a number of workers losing jobs, or moving into unemployment.

This is expressed formally in (3), reflecting in the first two factors of the equation the move from unemployment or migration to employment. These are processes complementary to those described in model (1) and (2). The rate at which the employed are separating from their jobs is $a_{4}$.

The death rates of the unemployed, employed and immigrants are $a_{3}, m_{3}$ and $b_{1}$.

Our system also captures the fact that government supports vacancies creation with the purpose to bring down unemployment and make better use of available human resources. There is no discrimination between natives and migrants in accessing new jobs, whether these are created by the market or through policy intervention. Both unemployment and migrant numbers are observed by the government and these numbers shape future intervention. Vacancies creation takes place with a delay, influenced by the process of making reliable data available, and the time needed for a policy response to be formulated. Equation (4) captures the active labour market policy supporting new vacancies, with different delays in the reaction to observed unemployment or migration.

The distributed time delay is introduced in the terms $\int_{0}^{\infty} k_{1}\left(d_{1}, s\right) U(t-s) d s$ and $\int_{0}^{\infty} k_{2}\left(d_{2}, s\right) M(t-s) d s$, where $k_{1}\left(d_{1}, s\right)$ with $d_{1}>0$ and $k_{2}\left(d_{2}, s\right)$ with $d_{2}>0$, are called "kernels" (Bernard et al. 2001). These represent density functions of the delays.

We also consider that there is variation over time in the jobs created by the market, depending on unemployment and implicit wage pressures associated with decreasing unemployment. This is expressed in Eq. (5).

The change over time in the number of jobs created by the market $P(t)$ is proportional to $U(t)$ and we add a jobs depreciation rate of $c_{2}$.

In view of the above considerations, the problem of unemployment reduction with migration and distributed delays may be written as follows:

$$
\begin{aligned}
\dot{U}(t)= & a_{1}-a_{2} U(t)(P(t)+V(t)-E(t))-a_{3} U(t)+a_{4} E(t)+a_{5} M(t), \\
\dot{M}(t)= & m_{1}-m_{2} M(t)(P(t)+V(t)-E(t))-\left(a_{5}+m_{3}\right) M(t), \\
\dot{E}(t)= & a_{2} U(t)(P(t)+V(t)-E(t))+m_{2} M(t)(P(t)+V(t)-E(t)) \\
& -\left(b_{1}+a_{4}\right) E(t), \\
\dot{V}(t)= & e_{1} \int_{0}^{\infty} k_{1}\left(d_{1}, s\right) U(t-s) d s+e_{3} \int_{0}^{\infty} k_{2}\left(d_{2}, s\right) M(t-s) d s-e_{2} V(t), \\
\dot{P}(t)= & c_{1} U(t)-c_{2} P(t) .
\end{aligned}
$$

where $a_{i}, i=1,2,3,4,5, b_{1}, c_{i}, i=1,2,3, d, d_{1}, d_{2}, e_{i}, i=1,2,3, m_{i}, i=1,2,3$ are real positive numbers.

In Eq. (4), the functions $k_{1}\left(d_{1}, s\right), k_{2}\left(d_{2}, s\right)$, are defined by $k_{i}\left(d_{i}, \cdot\right):[0, \infty) \rightarrow \mathbb{R}_{+}$, $i=1,2$ and satisfy the conditions:

1. $k_{i}\left(d_{i}, s\right) \Delta s$ represents the probability of the event of occurrence of delay between $s$ and $s+\Delta s$. That is, $k_{i}\left(d_{i}, s\right)$ satisfies the following: 


$$
\begin{aligned}
& k_{i}\left(d_{i}, s\right) \geq 0, \int_{0}^{\infty} k_{i}\left(d_{i}, s\right) d s=1, \\
& \quad \int_{0}^{\infty} s k_{i}\left(d_{i}, s\right) d s=\mathscr{E}\left(d_{i}\right), s \in[0, \infty), d_{i}>0, i=1,2
\end{aligned}
$$

where $\mathscr{E}\left(d_{i}\right)$ is the expectation of the distributed delay.

2. As the variable $s$ tends to infinity, it is rapidly decreasing or its support is compact.

In what follows, we consider the following types of expressions for $k_{i}\left(d_{i}, s\right)$ (Bernard et al. 2001):

1. The weak kernel

$$
k(d, s)=d e^{-d s}, d>0
$$

From (6) we have $\mathscr{E}(d)=\frac{1}{d}$.

2. The Dirac kernel

$$
k(\tau, s)=\delta(s-\tau), \tau>0,
$$

where $\delta(s-\tau)$ is the Dirac distribution. From (6) we have $\mathscr{E}(\tau)=\tau$.

If $k_{i}\left(d_{i}, s\right), i=1,2$, have the form (7), then Eq. (4) is given by

$$
\dot{V}(t)=e_{1} d_{1} \int_{0}^{\infty} e^{-d_{1} s} U(t-s) d s+e_{3} d_{2} \int_{0}^{\infty} e^{-d_{2} s} M(t-s) d s-e_{2} V(t) .
$$

If $k\left(\tau_{i}, s\right)$ are given by (8), then Eq. (4) is given by

$$
\dot{V}(t)=e_{1} U\left(t-\tau_{1}\right)+e_{3} M\left(t-\tau_{2}\right)-e_{2} V(t) .
$$

If $k_{1}\left(d_{1}, s\right)$ has the form (7) and $k_{2}\left(\tau_{2}, s\right)$ is given by (8), Eq. (4) is given by

$$
\dot{V}(t)=e_{1} d_{1} \int_{0}^{\infty} e^{-d_{1} s} U(t-s) d s+e_{3} M\left(t-\tau_{2}\right)-e_{2} V(t) .
$$

In the following we analyse the model (1)-(5) using the stability theory of the differential equation with distributed delay. We find the region of attraction given in the form of the following lemma:

\section{Lemma The set}

$$
\begin{aligned}
\Omega= & \left\{(U, M, E, V, P): 0 \leq U+M+E \leq \frac{a_{1}+m_{1}}{\delta},\right. \\
& \left.0 \leq V \leq \frac{\left(e_{1}+e_{3}\right)\left(a_{1}+m_{1}\right)}{\delta e_{2}}, 0 \leq P \leq \frac{c_{1}\left(a_{1}+m_{1}\right)}{\delta c_{2}}\right\}
\end{aligned}
$$

where $\delta=\min \left(a_{3}, b_{1}, m_{3}\right)$ is a region of attraction for the system (1)-(5) and it attracts all solutions initiating in the interior of the positive octant.

Proof See Appendix 1. 


\section{Equilibrium Analysis}

The non-negative equilibrium $E_{0}\left(x_{10}, x_{20}, x_{30}, x_{40}, x_{50}\right)$ is obtained by solving the following system:

$$
\begin{gathered}
a_{1}-a_{2} x_{1}\left(x_{5}+x_{4}-x_{3}\right)-a_{3} x_{1}+a_{4} x_{3}+a_{5} x_{2}=0 \\
m_{1}-m_{2} x_{2}\left(x_{5}+x_{4}-x_{3}\right)-\left(a_{5}+m_{3}\right) x_{2}=0 \\
a_{2} x_{1}\left(x_{5}+x_{4}-x_{3}\right)+m_{2} x_{2}\left(x_{5}+x_{4}-x_{3}\right)-\left(b_{1}+a_{4}\right) x_{3}=0 \\
e_{1} x_{1}+e_{3} x_{2}-e_{2} x_{4}=0 \\
c_{1} x_{1}-c_{2} x_{5}=0
\end{gathered}
$$

From (12)-(14), we obtain:

$$
a_{1}+m_{1}-a_{3} x_{1}-m_{3} x_{2}-b_{1} x_{3}=0
$$

From (15)-(16), we have:

$$
x_{4}=\frac{e_{1} x_{1}+e_{3} x_{3}}{e_{2}}, \quad x_{5}=\frac{c_{1} x_{1}}{c_{2}}, \quad x_{3}=\frac{a_{1}+m_{1}-a_{3} x_{1}-m_{3} x_{2}}{b_{1}} .
$$

From (18), we get:

$$
x_{4}+x_{5}-x_{3}=\frac{a b_{1} x_{1}+b b_{1} x_{2}-\left(a_{1}+m_{1}\right)}{b_{1}}
$$

where

$$
a=\frac{c_{1}}{c_{2}}+\frac{e_{1}}{e_{2}}+\frac{a_{3}}{b_{1}}, \quad b=\frac{e_{3}}{e_{2}}+\frac{m_{3}}{b_{1}} .
$$

From (19) and (12), we get:

$$
\begin{aligned}
& H_{\alpha}:=\alpha_{20} x_{1}^{2}+\alpha_{11} x_{1} x_{2}-\alpha_{10} x_{1}+\alpha_{01} x_{2}-\alpha_{00}=0, \\
& H_{\beta}:=\beta_{02} x_{2}^{2}+\beta_{11} x_{1} x_{2}-\beta_{01} x_{2}-\beta_{00}=0,
\end{aligned}
$$

where

$$
\begin{aligned}
& \alpha_{20}=a_{2} b_{1} a, \alpha_{11}=a_{2} b_{1} b, \\
& \alpha_{10}=a_{2}\left(a_{1}+m_{1}\right)-a_{3}\left(b_{1}+a_{4}\right), \\
& \alpha_{01}=a_{5} b_{1}-a_{4} m_{3}, \alpha_{00}=a_{1} b_{1}+a_{4}\left(a_{1}+m_{1}\right), \\
& \beta_{02}=m_{2} b_{1} b, \beta_{11}=m_{2} b_{1} a, \\
& \beta_{01}=m_{2}\left(a_{1}+m_{1}\right)-b_{1}\left(a_{3}+a_{5}\right), \\
& \beta_{00}=m_{1} b_{1} .
\end{aligned}
$$


Equations (21) and (22) represent the equations of two hyperboles. The hyperbole $H_{\alpha}$ has the center $C_{\alpha}\left(\frac{\alpha_{01}}{\alpha_{11}}, \frac{\alpha_{11} \alpha_{10}-2 \alpha_{20} \alpha_{01}}{\alpha_{11}^{2}}\right)$ and the asymptotes $x_{1}=\frac{\alpha_{01}}{\alpha_{11}}$ and $x_{2}+\frac{\alpha_{11} \alpha_{10}-2 \alpha_{20} \alpha_{01}}{\alpha_{11}^{2}}=-\frac{a}{b}\left(x_{1}-\frac{\alpha_{01}}{\alpha_{11}}\right)$.

The hyperbola $H_{\beta}$ has the center $C_{\beta}\left(\frac{\beta_{01}}{\beta_{11}}, 0\right)$ and the asymptotes $x_{1}=0, x_{2}=$ $-\frac{a}{b}\left(x_{1}-\frac{\beta_{01}}{\beta_{11}}\right)$.

A necessary condition, as the hyperboles $H_{\alpha}, H_{\beta}$ have a common point with positive coordinates, is that the coordinates of the centers are positive and $\frac{a}{b}>1$. Thus, we have the conditions:

$$
a>b, b \alpha_{10}>2 a \alpha_{01}, m_{2} a \alpha_{01}<a_{2} b \beta_{01} .
$$

Now, we determine the equation which determines the coordinate $x_{10}$ of the intersection point.

From (21), we get:

$$
x_{2}=\frac{\alpha_{00}+\alpha_{10} x_{1}-\alpha_{20} x_{1}^{2}}{\alpha_{11} x_{1}-\alpha_{01}}
$$

From (25), (22), we have:

$$
\gamma_{3} x_{1}^{3}+\gamma_{2} x_{1}^{2}+\gamma_{1} x_{1}+\gamma_{0}=0
$$

with

$$
\begin{aligned}
\gamma_{3}= & \alpha_{10} \alpha_{11} \beta_{11}-\alpha_{20}\left(\beta_{11} \alpha_{01}+\alpha_{11} \beta_{01}\right), \\
\gamma_{2}= & \alpha_{00} \alpha_{11} \beta_{11}-\beta_{02} \alpha_{10}^{2}+\alpha_{10}\left(\beta_{11} \alpha_{01}+\alpha_{11} \beta_{01}\right) \\
& +\alpha_{20} \alpha_{01} \beta_{01}+\beta_{00} \alpha_{11}^{2}, \\
\gamma_{1}= & -2 \beta_{02} \alpha_{00} \alpha_{10}-\alpha_{01} \alpha_{10} \beta_{01} \\
& +\alpha_{00}\left(\beta_{11} \alpha_{01}+\alpha_{11} \beta_{01}\right)-2 \beta_{00} \alpha_{11} \alpha_{01}, \\
\gamma_{0}= & \beta_{00} \alpha_{01}^{2}-\alpha_{00}\left(\beta_{02} \alpha_{00}+\beta_{01} \alpha_{01}\right)
\end{aligned}
$$

Proposition 1 If

$$
\begin{aligned}
a & >b, b \alpha_{10}>2 a \alpha_{01}, m_{2} a \alpha_{01}<a_{2} b \beta_{01} \\
\gamma_{3} & >0, \gamma_{2}>0, \gamma_{1}<0, \gamma_{0}<0,
\end{aligned}
$$

then the non-negative equilibrium $E_{0}$ has the coordinates:

$$
\begin{aligned}
& x_{20}=\frac{\alpha_{00}+\alpha_{10} x_{10}-\alpha_{20} x_{10}^{2}}{\alpha_{11} x_{10}-\alpha_{01}}, \\
& x_{30}=\frac{a_{1}+m_{1}-a_{3} x_{10}-m_{3} x_{20}}{b_{1}},
\end{aligned}
$$




$$
x_{40}=\frac{e_{1} x_{10}+e_{3} x_{20}}{e_{2}}, x_{50}=\frac{c_{1} x_{10}}{c_{2}},
$$

where $x_{10}$ is the positive solution of Eq. (26).

\section{Proof See Appendix 2.}

Using the transformation $u_{1}(t)=U(t)-x_{10}, u_{2}(t)=M(t)-x_{20}, u_{3}(t)=$ $E(t)-x_{30}, u_{4}(t)=V(t)-x_{40}, u_{5}(t)=P(t)-x_{50}$, system (1)-(5) becomes:

$$
\begin{aligned}
\dot{u}_{1}(t)= & -\left(\alpha_{3}+a_{3}\right) u_{1}(t)+a_{5} u_{2}(t)+\left(\alpha_{1}+a_{4}\right) u_{3}(t)-\alpha_{1} u_{4}(t)-\alpha_{1} u_{5}(t) \\
& -a_{2} u_{1}(t)\left(u_{5}(t)+u_{4}(t)-u_{3}(t)\right) \\
\dot{u}_{2}(t)=- & \left(\alpha_{4}+a_{5}+m_{3}\right) u_{2}(t)+\alpha_{2} u_{3}(t)-\alpha_{2} u_{4}(t)-\alpha_{2} u_{5}(t) \\
& -m_{2} u_{2}(t)\left(u_{5}(t)+u_{4}(t)-u_{3}(t)\right), \\
\dot{u}_{3}(t)= & \alpha_{3} u_{1}(t)-\alpha_{4} u_{2}(t)-\left(\alpha_{1}+\alpha_{2}+b_{1}+a_{4}\right) u_{3}(t)+\left(\alpha_{1}+\alpha_{2}\right) u_{4}(t) \\
& +\left(\alpha_{1}+\alpha_{2}\right) u_{5}(t)+a_{2} u_{1}(t)\left(u_{5}(t)+u_{4}(t)\right. \\
& \left.-u_{3}(t)\right)+m_{2} u_{2}(t)\left(u_{5}(t)+u_{4}(t)-u_{3}(t)\right) \\
\dot{u}_{4}(t)= & e_{1} \int_{0}^{\infty} k_{1}\left(d_{1}, s\right) u_{1}(t-s) d s+e_{3} \int_{0}^{\infty} k_{2}\left(d_{2}, s\right) u_{2}(t-s) d s-e_{2} u_{4}(t), \\
\dot{u}_{5}(t)= & c_{1} u_{1}(t)-c_{2} u_{5}(t)
\end{aligned}
$$

where

$$
\begin{aligned}
& \alpha_{1}=a_{2} x_{10}, \alpha_{2}=m_{2} x_{20}, \alpha_{3}=a_{2}\left(x_{50}+x_{40}-x_{30}\right), \\
& \alpha_{4}=m_{2}\left(x_{50}+x_{40}-x_{30}\right) .
\end{aligned}
$$

The system (30) is given by:

$$
\dot{u}(t)=A u(t)+\int_{0}^{\infty} K\left(d_{1}, d_{2}, s\right) u(t-s) d s+F(u(t))
$$

where

$$
\begin{aligned}
u(t) & =\left(u_{1}(t), \quad u_{2}(t), \quad u_{3}(t), \quad u_{4}(t), \quad u_{5}(t)\right)^{T}, \\
A & =\left(\begin{array}{ccccc}
a_{11} & a_{12} & a_{13} & -\alpha_{1} & -\alpha_{1} \\
0 & a_{22} & \alpha_{2} & -\alpha_{2} & -\alpha_{2} \\
\alpha_{3} & -\alpha_{4} & 0 & a_{34} & a_{35} \\
0 & 0 & 0 & -e_{2} & 0 \\
c_{1} & 0 & 0 & 0 & -c_{2}
\end{array}\right)
\end{aligned}
$$

where

$$
\begin{aligned}
& a_{11}=-\alpha_{3}-a_{3}, a_{12}=a_{5}, a_{22}=-\alpha_{4}-a_{5}-m_{3}, \\
& a_{13}=\alpha_{1}+a_{4}, a_{33}=-\alpha_{1}-\alpha_{2}-b_{1}-a_{4}, a_{34}=\alpha_{1}+\alpha_{2}, a_{35}=\alpha_{1}+\alpha_{2},
\end{aligned}
$$




$$
\begin{gathered}
K\left(d_{1}, d_{2}, s\right)=\left(\begin{array}{ccccc}
0 & 0 & 0 & 0 & 0 \\
0 & 0 & 0 & 0 & 0 \\
0 & 0 & 0 & 0 & 0 \\
e_{1} k_{1}\left(d_{1}, s\right) & e_{3} k_{2}\left(d_{2}, s\right) & 0 & 0 & 0 \\
0 & 0 & 0 & 0 & 0
\end{array}\right) \\
F(u(t))=\left(F^{1}(u(t)), F^{2}(u(t)), F^{3}(u(t)), 0,0\right)^{T} \text { with } \\
F^{1}(u(t))=-a_{2} u_{1}(t)\left(u_{5}(t)+u_{4}(t)-u_{3}(t)\right), \\
F^{2}(u(t))=-m_{2} u_{2}(t)\left(u_{5}(t)+u_{4}(t)-u_{3}(t)\right), \\
F^{3}(u(t))=-F^{1}(u(t))-F^{2}(u(t)) .
\end{gathered}
$$

\section{Stability Analysis}

In this section, we study the local stability behaviour of the non-negative equilibrium $E_{0}$, in both the cases of no distributed delay and distributed delay.

Using the matrices $A$ and $K\left(d_{1}, d_{2}, s\right)$ from (34) and (35), the characteristic equation of the linearised system (30) is as follows:

$$
Q_{3}(\lambda)+Q_{1}(\lambda) \int_{0}^{\infty} k_{1}\left(d_{1}, s\right) e^{-\lambda s} d s+Q_{2}(\lambda) \int_{0}^{\infty} k_{2}\left(d_{2}, s\right) e^{-\lambda s} d s=0,
$$

where

where

$$
\begin{aligned}
& Q_{3}(\lambda)=\lambda^{5}+q_{34} \lambda^{4}+q_{33} \lambda^{3}+q_{32} \lambda^{2}+q_{31} \lambda+q_{30}, \\
& Q_{2}(\lambda)=q_{23} \lambda^{3}+q_{22} \lambda^{2}+q_{21} \lambda+q_{20}, \\
& Q_{1}(\lambda)=q_{13} \lambda^{3}+q_{12} \lambda^{2}+q_{11} \lambda+q_{10},
\end{aligned}
$$

$$
\begin{aligned}
q_{34}= & c_{2}+e_{2}-a_{11}-a_{22}-a_{33}, \\
q_{33}= & c_{1} \alpha_{1}+e_{2} c_{2}-\left(c_{2}+e_{2}\right)\left(a_{11}+a_{22}+a_{33}\right)+a_{22} a_{33}+a_{11}\left(a_{22}+a_{33}\right) \\
& -a_{13} \alpha_{3}+\alpha_{2} \alpha_{4} \\
q_{32}= & e_{1} e_{2} \alpha_{1}-c_{1}\left(\alpha_{1}\left(a_{22}+a_{33}\right)+a_{13} a_{35}-\alpha_{2} a_{12}\right)-e_{2} c_{2}\left(a_{11}+a_{22}+a_{33}\right) \\
& +\left(c_{2}+e_{2}\right)\left(a_{22} a_{33}+a_{11}\left(a_{22}+a_{33}\right)-a_{13} \alpha_{3}+\alpha_{2} \alpha_{4}\right) \\
& -a_{11} a_{22} a_{33}-\alpha_{2} \alpha_{3} a_{12}+\alpha_{3} a_{13} a_{22} \\
& -\alpha_{2} \alpha_{4} a_{11}, \\
q_{31}= & -e_{1} e_{2}\left(\alpha_{1}\left(a_{22}+a_{33}\right)+a_{13} a_{35}-\alpha_{2} a_{12}\right)+c_{1}\left(-a_{12} a_{35} \alpha_{2}-\alpha_{2} \alpha_{4} a_{13}\right. \\
& \left.+\alpha_{1} a_{22} a_{33}+\alpha_{1} \alpha_{2} \alpha_{4}+a_{13} a_{22} a_{35}-\alpha_{2} a_{12} a_{33}\right) \\
& +e_{2} c_{2}\left(a_{22} a_{33}+a_{11}\left(a_{22}+a_{33}\right)-a_{13} \alpha_{3}\right. \\
& \left.+\alpha_{2} \alpha_{4}\right)+\left(c_{2}+e_{2}\right)\left(-a_{11} a_{22} a_{33}-\alpha_{2} \alpha_{3} a_{12}+\alpha_{3} a_{13} a_{22}-\alpha_{2} \alpha_{4} a_{11}\right) \\
q_{30}= & c_{1} c_{2}\left(-a_{12} a_{32} \alpha_{2}-\alpha_{2} \alpha_{4} a_{13}+\alpha_{1} a_{22} a_{33}+\alpha_{1} \alpha_{2} \alpha_{4}+a_{13} a_{22} a_{35}-\alpha_{2} a_{12} a_{33}\right) \\
& +e_{2} c_{2}\left(-a_{11} a_{22} a_{33}-\alpha_{2} \alpha_{3} a_{12}+\alpha_{3} a_{13} a_{22}-\alpha_{2} \alpha_{4} a_{11}\right), \\
q_{23}= & -e_{3} d_{2},
\end{aligned}
$$




$$
\begin{aligned}
q_{22}= & -e_{3} c_{2} \alpha_{2}+e_{3} \alpha_{2}\left(a_{11}+a_{33}\right)+e_{3} \alpha_{2} a_{34}, \\
q_{21}= & e_{3} c_{2} \alpha_{2}\left(a_{11}+a_{33}+\alpha_{2} a_{34}\right)-e_{3} \alpha_{2} a_{11} a_{34}+e_{3} \alpha_{2} \alpha_{3} a_{13}-e_{3} \alpha_{1} \alpha_{2} \alpha_{3} \\
& -e_{2} \alpha_{2} a_{11} a_{33}, \\
q_{20}= & e_{3} c_{1}\left(\alpha_{2} a_{13}-\alpha_{1} \alpha_{2}\right)\left(a_{35}-a_{34}\right)+e_{3} c_{2}\left(-\alpha_{2} a_{11} a_{34}+\alpha_{2} \alpha_{3} a_{13}-\alpha_{1} \alpha_{2} \alpha_{3}\right. \\
& \left.-\alpha_{2} a_{11} a_{33}\right) \\
q_{13}= & e_{1} \alpha_{1}, \\
q_{12}= & \alpha_{1} e_{1} c_{2}-e_{1}\left(\alpha_{1}\left(a_{22}+a_{33}\right)+a_{13} a_{34}-\alpha_{2} a_{12}\right) \\
q_{11}= & -e_{1} c_{2}\left(\alpha_{1}\left(a_{22}+a_{33}\right)+a_{13} a_{34}-\alpha_{2} a_{12}\right)+e_{1}\left(-a_{12} a_{34} \alpha_{2}-a_{13} \alpha_{2} \alpha_{4}\right. \\
& \left.+\alpha_{1} a_{22} a_{33}+\alpha_{1} \alpha_{2} \alpha_{4}+a_{13} a_{34} a_{22}-\alpha_{2} a_{12} a_{33}\right) \\
q_{10}= & e_{2} c_{2}\left(-a_{12} a_{34} \alpha_{2}-a_{13} \alpha_{2} \alpha_{4}+\alpha_{1} a_{22} a_{33}+\alpha_{1} \alpha_{2} \alpha_{4}+a_{13} a_{34} a_{22}-\alpha_{2} a_{12} a_{33}\right) .
\end{aligned}
$$

Proposition 2 If there is no effect of distributed delay, that is when $\mathscr{E}=0$, the equilibrium point $E_{0}$ given by (29) is locally asymptotically stable if and only if the following conditions hold:

$$
\begin{aligned}
& A_{1}>0, A_{5}>0, A_{1} A_{2}-A_{3}>0, \\
& \quad A_{1} A_{2} A_{3}-A_{3}^{2}-A_{1}^{2} A_{4}>0, \\
& \left(A_{3} A_{4}-A_{2} A_{5}\right)\left(A_{1} A_{2}-A_{3}\right)-\left(A_{1} A_{4}-A_{5}\right)^{2}>0,
\end{aligned}
$$

where $A_{1}=q_{34}, A_{2}=q_{13}+q_{23}+q_{33}, A_{3}=q_{12}+q_{22}+q_{32}, A_{4}=q_{11}+q_{21}+q_{31}$, $A_{5}=q_{10}+q_{20}+q_{30}$.

Proof See Appendix 3.

We analyse (37) in the case when the expectation of distributed delay is $\mathscr{E}_{i}\left(d_{i}\right) \neq 0$, $d_{i}>0, i=1,2, d_{1}=d_{2}$.

Theorem 1 Suppose that $q_{30}-q_{20}-q_{01}<0$ and the conditions from (40) hold. Then the characteristic equation

$$
\begin{aligned}
& \lambda^{5}+q_{34} \lambda^{4}+q_{33} \lambda^{3}+q_{32} \lambda^{2}+q_{31} \lambda+q_{30} \\
& +\left(q_{13}+q_{23}\right) \lambda^{3}+\left(q_{12}+q_{22}\right) \lambda^{2}+\left(q_{11}+q_{21}\right) \lambda \\
& \quad+\left(q_{10}+q_{20}\right) \int_{0}^{\infty} k\left(d_{1}, s\right) e^{-\lambda s} d s=0
\end{aligned}
$$

has a simple pair of conjugate purely imaginary roots $\pm i \omega$ with some expectation $\mathscr{E}_{1}\left(d_{1}\right)$.

Proof See Appendix 4.

1. If both kernels are Dirac $k_{i}\left(\tau_{i}, s\right)=\delta\left(s-\tau_{i}\right), \tau_{i}>0, i=1,2$ with $\tau_{1}=\tau_{2}=\tau$, the characteristic Eq. (41) is

$$
\begin{aligned}
& \lambda^{5}+q_{34} \lambda^{4}+q_{33} \lambda^{3}+q_{32} \lambda^{2}+q_{31} \lambda+q_{30} \\
& \quad+\left(q_{13}+q_{23}\right) \lambda^{3}+\left(q_{12}+q_{22}\right) \lambda^{2}+\left(q_{11}+q_{21}\right) \lambda+\left(q_{10}+q_{20}\right) e^{-\lambda \tau}=0 .
\end{aligned}
$$

Using the method from Mircea et al. (2011) we have: 
Theorem 2 Suppose that the conditions (40) and $q_{30}-q_{20}-q_{10}<0$ hold. Then, Eq. (42) has a simple pair of conjugate purely imaginary roots $\pm i \omega_{0}$, where $\omega_{0}$ is a positive root of Eq. (71). For $\omega_{0}$, we have:

$$
\begin{aligned}
\tau_{0}= & \frac{1}{\omega_{0}} \arccos \left(\frac{\left(q_{32} \omega_{0}^{2}-q_{34} q_{30} \omega_{0}^{4}\right)\left(q_{10}+q_{20}-\left(q_{11}+q_{22}\right) \omega_{0}^{2}\right)}{\left(q_{10}+q_{20}-\left(q_{12}+q_{22}\right) \omega_{0}^{2}\right)^{2}\left(\left(q_{11}+q_{21}\right) \omega_{0}-\left(q_{13}+q_{23}\right) \omega_{0}^{3}\right)^{2}}\right. \\
+ & \left.\frac{\left(\omega_{0}^{5}+q_{33} \omega_{0}^{3}-q_{31} \omega_{0}\right)\left(\left(q_{11}+q_{21}\right) \omega_{0}-\left(q_{12}+q_{23}\right) \omega_{0}^{3}\right)}{\left(q_{10}+q_{20}-\left(q_{12}+q_{22}\right) \omega_{0}^{2}\right)^{2}+\left(\left(q_{11}+q_{21}\right) \omega_{0}-\left(q_{13}+q_{23}\right) \omega_{0}^{3}\right)^{2}}+2 n \pi\right), \\
& \quad n=0,1,2, \ldots
\end{aligned}
$$

Taking into account that $\int_{0}^{\infty} k_{1}(\tau, s) e^{-\lambda s} d s=e^{-\lambda \tau}$ and using Theorem 1 we obtain Theorem 2.

From Theorem 2, we obtain:

Theorem 3 Suppose the conditions (40) and $q_{30}-q_{20}-q_{10}<0$ hold. Then, we have:

1. If $\tau \in\left[0, \tau_{0}\right)$, then the equilibrium point $E_{0}$ is locally asymptotically stable;

2. If the condition

$$
\begin{aligned}
& \operatorname{Re}\left(\frac{\left(5 \lambda^{4}+4 q_{34} \lambda^{3}+3 q_{33} \lambda^{2}+2 q_{32} \lambda+q_{31}\right) e^{\lambda \tau}}{\lambda\left(\left(q_{13}+q_{23}\right) \lambda^{3}+\left(q_{12}+q_{22}\right) \lambda^{2}+\left(q_{11}+q_{21}\right) \lambda+q_{10}+q_{20}\right)}\right. \\
& \left.\quad+\frac{3\left(q_{13}+q_{23}\right) \lambda^{2}+2\left(q_{12}+q_{22}\right) \lambda+q_{11}+q_{21}}{\lambda\left(\left(q_{13}+q_{23}\right) \lambda^{3}+\left(q_{12}+q_{22}\right) \lambda^{2}+\left(q_{11}+q_{21}\right) \lambda+q_{10}+q_{20}\right)}\right)\left.\right|_{\lambda=i \omega_{0}, \tau=\tau_{0}} \neq 0
\end{aligned}
$$

holds, then in (32) the Hopf bifurcation occurs when $\tau=\tau_{0}$.

\section{Proof See Appendix 5.}

Remark If Eq. (71) has $r_{0}=q_{30}^{2}-\left(q_{10}+q_{20}\right)^{2}>0$ and does not have positive roots, then the equilibrium point $E_{0}$ is locally asymptotically stable for all $\tau \geq 0$.

2. If both kernels are weak $k_{i}\left(d_{i}, s\right)=d_{i} e^{-d_{i} s}, d_{1}=d_{2}>0$, then the characteristic equation (41) becomes:

$$
\lambda^{6}+A_{1} \lambda^{5}+A_{2} \lambda^{4}+A_{3} \lambda^{3}+A_{4} \lambda^{2}+A_{5} \lambda+A_{6}=0,
$$

where

$$
\begin{aligned}
& A_{1}=q_{34}+d_{1}, A_{2}=q_{33}+d_{1} q_{34}, \\
& A_{3}=q_{32}+d_{1}\left(q_{13}+q_{23}+q_{33}\right), \\
& A_{4}=q_{31}+d_{1}\left(q_{12}+q_{22}+q_{32}\right), \\
& A_{5}=q_{30}+d_{1}\left(q_{11}+q_{21}+q_{31}\right), A_{6}=d_{1}\left(q_{10}+q_{20}+q_{30}\right) .
\end{aligned}
$$


In what follows we consider:

$$
\begin{aligned}
D_{1}\left(d_{1}\right):= & A_{1} A_{2}-A_{3}, \\
D_{2}\left(d_{1}\right):= & A_{1} A_{2} A_{3}+A_{1} A_{5}-A_{3}^{2}-A_{4} A_{1}^{2}, \\
D_{3}\left(d_{1}\right):= & A_{1}\left(A_{1} A_{3} A_{4}+A_{1} A_{2} A_{6}+A_{4} A_{5}\right. \\
& \left.-A_{3} A_{6}-A_{1} A_{4}^{2}-A_{5} A_{2}^{2}\right) \\
& -\left(A_{3}^{2} A_{4}+A_{5}^{2}-A_{1} A_{4} A_{5}-A_{2} A_{3} A_{5}\right), \\
D_{4}\left(d_{1}\right):= & A_{5} D_{3}\left(d_{1}\right)-A_{6}\left|\begin{array}{cccc}
A_{1} & 1 & 0 & 0 \\
A_{3} & A_{2} & A_{1} & 0 \\
A_{5} & A_{4} & A_{3} & A_{1} \\
0 & A_{6} & A_{5} & A_{3}
\end{array}\right|, \\
D_{5}\left(d_{1}\right):= & A_{6} D_{4}\left(d_{1}\right) .
\end{aligned}
$$

Proposition 4 If the conditions $D_{1}\left(d_{1}\right)>0, D_{2}\left(d_{1}\right)>0, D_{3}\left(d_{1}\right)>0, D_{4}\left(d_{1}\right)>0$, $D_{5}\left(d_{1}\right)>0$ hold, for any $d_{1}>0$, the equilibrium point $E_{0}$ of system (1)-(5) is locally asymptotically stable.

The proof follows using the Routh-Hurwicz criteria for (45).

In what follows we consider the case $k_{1}\left(\tau_{1}, s\right)=\delta\left(s-\tau_{1}\right), k_{2}\left(\tau_{2}, s\right)=\delta\left(s-\tau_{2}\right)$, with $\tau_{1} \neq 0, \tau_{2} \neq 0$.

Equation (37) becomes:

$$
Q_{3}(\lambda)+Q_{1}(\lambda) e^{-\lambda \tau_{1}}+Q_{2}(\lambda) e^{-\lambda \tau_{2}}=0,
$$

where $Q_{1}, Q_{2}, Q_{3}$ are given by (38).

For analysing Eq. (48) we consider the following subcases:

Case 1. $\tau_{1} \neq 0, \tau_{2}=0$

In this case, eq. (48) becomes:

$$
Q_{3}(\lambda)+Q_{2}(\lambda)+Q_{1}(\lambda) e^{-\lambda \tau_{1}}=0 .
$$

Considering $\lambda=i \omega_{1}$ in (49) and separating the real and the imaginary parts we have:

$$
\begin{aligned}
& q_{34} \omega_{1}^{4}-\left(q_{22}+q_{32}\right) \omega_{1}^{2}+q_{20}+q_{30} \\
& \quad=\left(q_{12} \omega_{1}^{2}-q_{10}\right) \cos \left(\omega_{1} \tau_{1}\right)+\left(q_{13} \omega_{1}^{3}-q_{11} \omega_{1}\right) \sin \left(\omega_{1} \tau_{1}\right), \\
& \omega_{1}^{5}-\left(q_{23}+q_{33}\right) \omega_{1}^{3}+\left(q_{21}+q_{31}\right) \omega_{1} \\
& \quad=\left(q_{13} \omega_{1}^{3}-q_{11} \omega_{1}\right) \cos \left(\omega_{1} \tau_{1}\right)-\left(q_{12} \omega_{1}^{2}-q_{10}\right) \sin \left(\omega_{1} \tau_{1}\right) .
\end{aligned}
$$

From (50), we have:

$$
\omega_{1}^{10}+p_{8} \omega_{1}^{8}+p_{6} \omega_{1}^{6}+p_{4} \omega_{1}^{4}+p_{2} \omega_{1}^{2}+p_{0}=0
$$

where

$$
p_{8}=q_{34}^{2}-2\left(q_{23}+q_{23}\right) \text {. }
$$




$$
\begin{aligned}
& p_{6}=\left(q_{23}+q_{33}\right)^{2}+2\left(q_{21}+q_{31}\right)-2 q_{34}\left(q_{22}+q_{32}\right)-q_{13}^{2}, \\
& p_{4}=\left(q_{22}+q_{32}\right)^{2}+2 q_{34}\left(q_{20}+q_{30}\right)-2\left(q_{23}+q_{33}\right)\left(q_{21}+q_{31}\right)-q_{12}^{2}+2 q_{13} q_{11}, \\
& p_{2}=\left(q_{21}+q_{31}\right)^{2}-2\left(q_{22}+q_{32}\right)\left(q_{20}+q_{30}\right)+2 q_{12} q_{10}, \\
& p_{0}=\left(q_{20}+q_{30}\right)^{2}-q_{10}^{2} .
\end{aligned}
$$

Proposition 5 Suppose that the conditions (40) and $q_{20}+q_{30}-q_{10}<0$ hold. Then, Eq. (49) has a simple pair of conjugate purely imaginary roots $\pm i \omega_{01}$, where $\omega_{01}$ is a positive root of Eq. (51). For $\omega_{01}$ we have:

$$
\begin{aligned}
\tau_{01}= & \frac{1}{\omega_{01}}\left\{\operatorname { a r c c o s } \left[\left(q_{13} \omega_{01}^{2}-q_{11} \omega_{01}\right)\left(q_{34} \omega_{01}^{3}-\left(q_{22}+q_{32}\right) \omega_{01}^{2}+q_{20}+q_{30}\right)\right.\right. \\
& +\left(q_{12} \omega_{01}^{2}-q_{10}\right)\left(\omega_{01}^{5}-\left(q_{23}+q_{33}\right) \omega_{01}^{3}+\left(q_{21}+q_{31}\right) \omega_{01}\right)\left(\left(q_{12} \omega_{01}^{2}-q_{10}\right)^{2}\right. \\
& \left.\left.\left.+\left(q_{13} \omega_{01}^{3}-q_{11} \omega_{01}\right)^{2}\right)\right]\right\}+2 n \pi, n=0,1, \ldots
\end{aligned}
$$

From Proposition 5, we obtain:

Proposition 6 Suppose conditions (40) and $q_{20}+q_{30}-q_{10}<0$ hold. Then, we have:

1. If $\tau_{1} \in\left[0, \tau_{01}\right]$, then the equilibrium point $E_{0}$ is locally asymptotically stable;

2. If the condition

$$
\begin{aligned}
& \operatorname{Re}\left(\frac{\left(5 \lambda^{4}+4 q_{34} \lambda^{3}+3\left(q_{23}+q_{33}\right) \lambda^{2}+2\left(q_{22}+q_{32}\right) \lambda+q_{21}+q_{31}\right) e^{\lambda \tau_{1}}}{\lambda\left(q_{13} \lambda^{3}+q_{12} \lambda^{2}+q_{11} \lambda+q_{10}\right)}\right. \\
& \left.\quad+\frac{3 \lambda^{2}+2 q_{12} \lambda+q_{11}}{\lambda\left(q_{13} \lambda^{3}+q_{12} \lambda^{2}+q_{11} \lambda+q_{10}\right)}\right)\left.\right|_{\lambda=i \omega_{01}, \tau_{1}=\tau_{10}} \neq 0
\end{aligned}
$$

holds,

then in (49) the Hopf bifurcation occurs when $\tau_{1}=\tau_{01}$.

The proof can be done in a similar way as for Theorem 3 .

Case 2. Let $\tau_{1}^{*} \in\left[0, \tau_{10}\right)$ fixed and $\tau_{2} \neq 0$. We determine the value $\tau_{20}>0, \tau_{20}\left(\tau_{1}^{*}\right)$ so that equation:

$$
Q_{3}(\lambda)+Q_{1}(\lambda) e^{-\lambda \tau_{1}^{*}}+Q_{2}(\lambda) e^{-\lambda \tau_{2}}=0
$$

admits the roots $\lambda_{2}\left(\tau_{2}\right)= \pm i \omega_{2}\left(\tau_{2}\left(\tau_{1}\right)\right)$.

We use the following notation:

$$
\begin{aligned}
A\left(\omega_{2}\right)= & \left(q_{34} \omega_{2}^{4}-q_{32} \omega_{2}^{2}+q_{30}\right)\left(q_{10}-q_{12} \omega_{2}^{2}\right) \\
& -\left(\omega_{2}^{5}+q_{33} \omega_{2}^{3}-q_{31} \omega_{2}\right)\left(q_{11} \omega_{2}-q_{13} \omega_{2}^{3}\right), \\
B\left(\omega_{2}\right)= & \left(q_{34} \omega_{2}^{4}-q_{32} \omega_{2}^{2}+q_{30}\right)\left(q_{11} \omega_{2}-q_{13} \omega_{2}^{3}\right)
\end{aligned}
$$




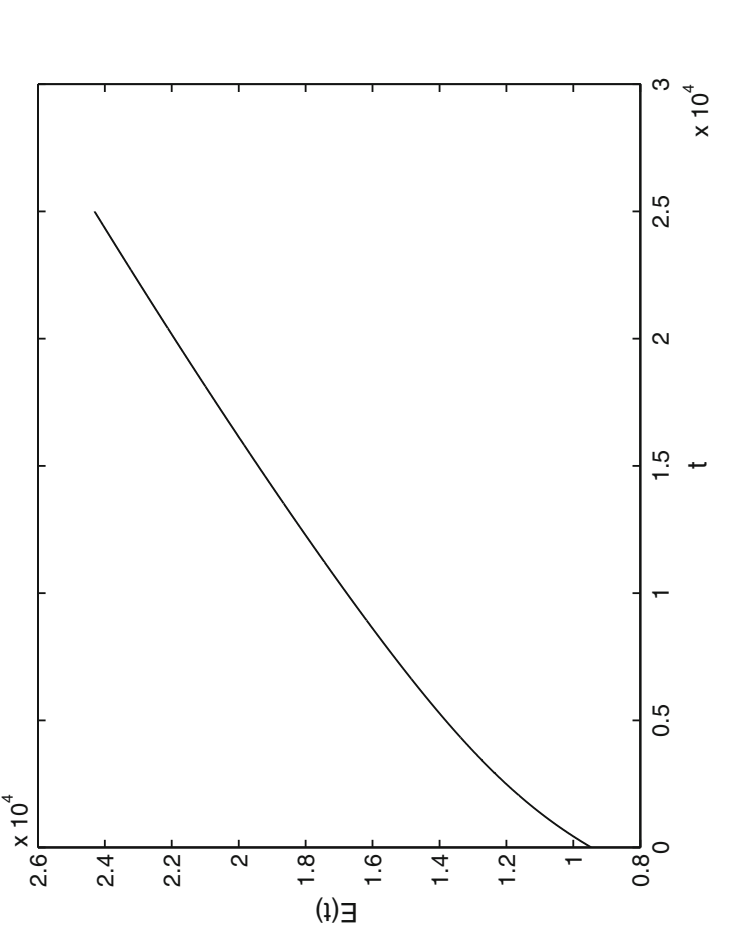

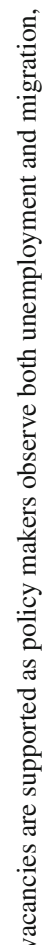

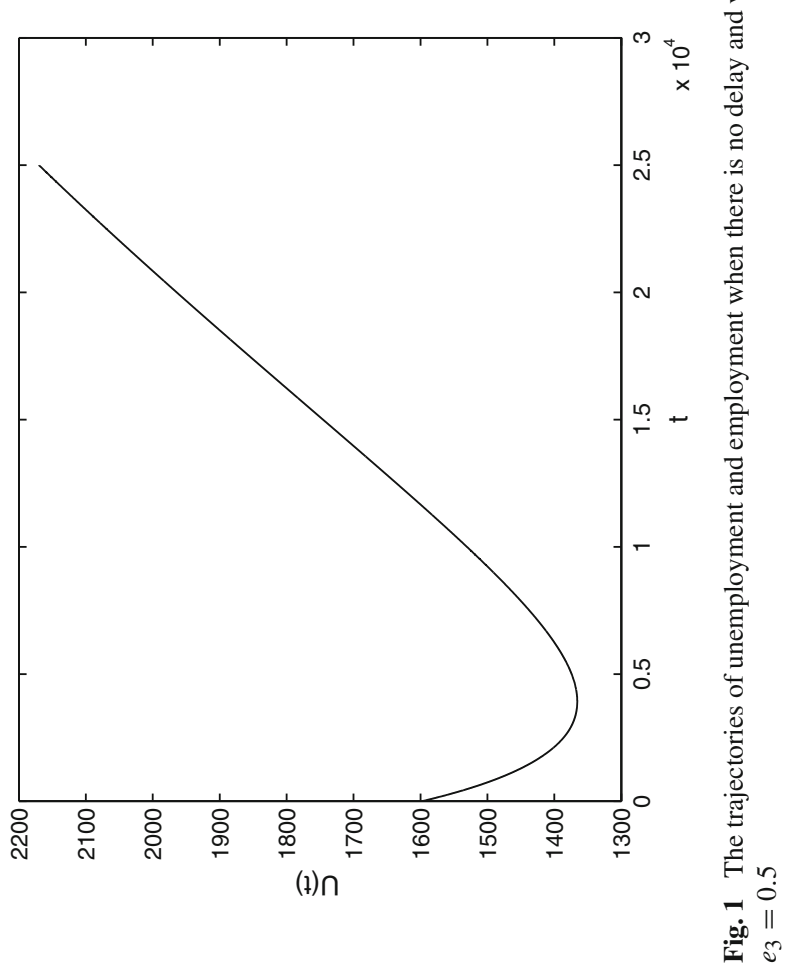




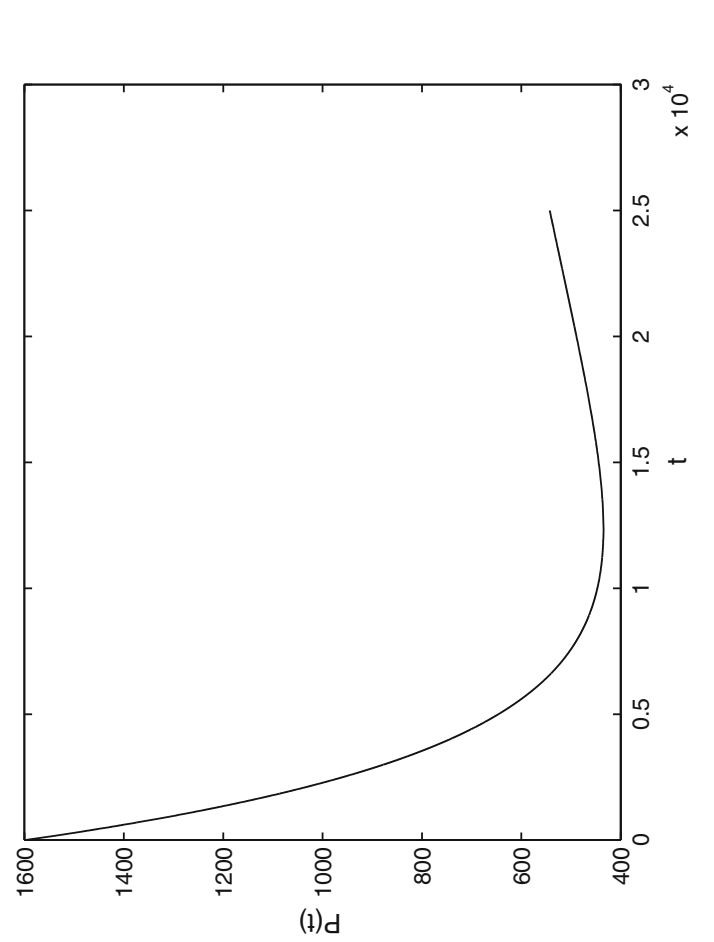

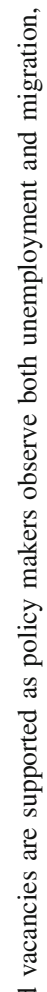

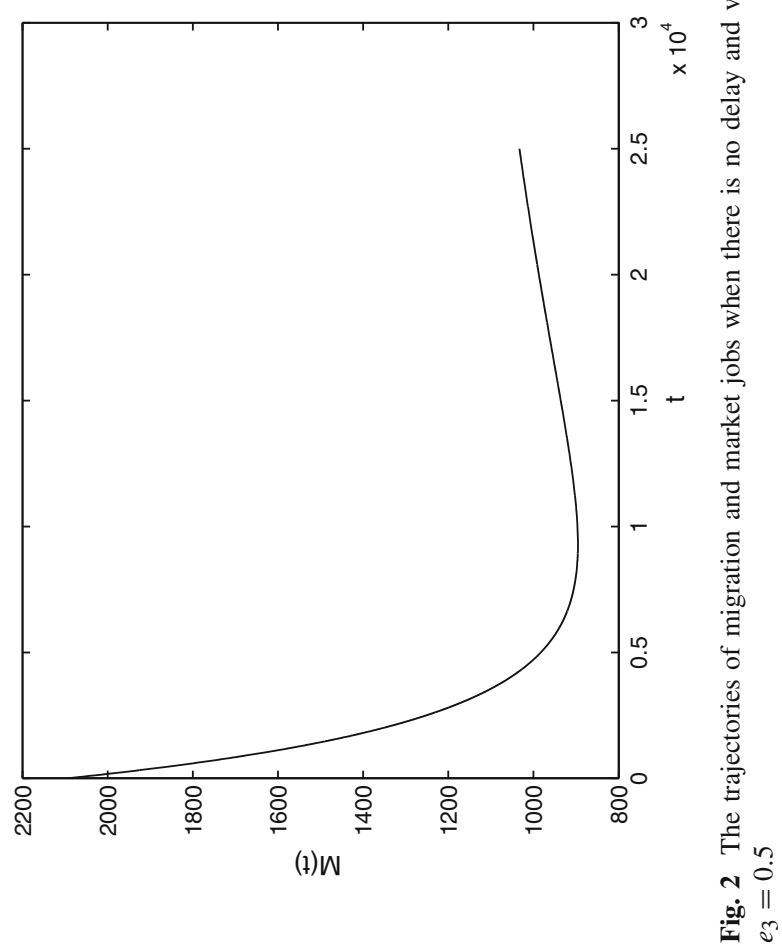




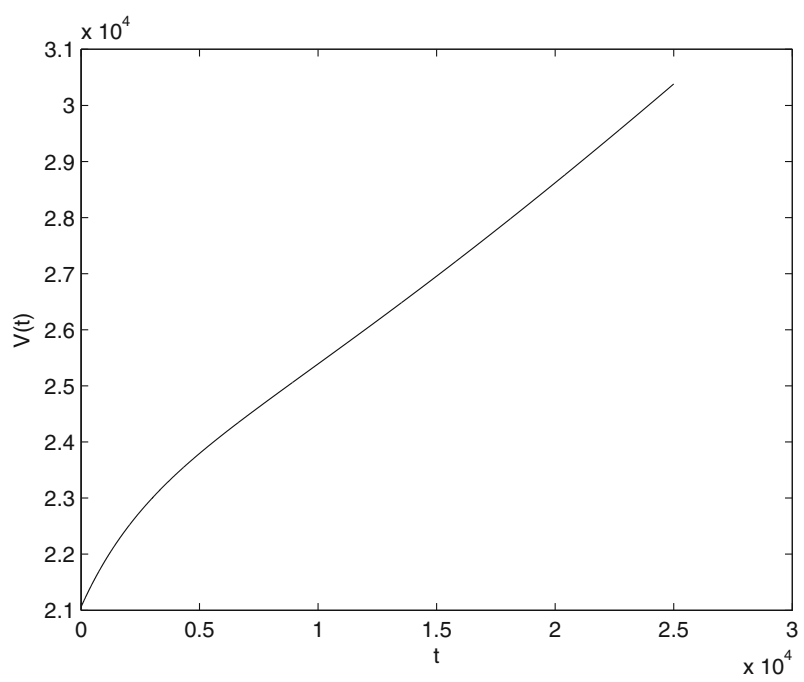

Fig. 3 The trajectories of vacancies created through government intervention when there is no delay and vacancies are supported as policy makers observe both unemployment and migration, $e_{3}=0.5$

$$
\begin{aligned}
& +\left(\omega_{2}^{5}+q_{33} \omega_{2}^{3}-q_{1} \omega_{2}\right)\left(q_{10}-q_{12} \omega_{2}^{2}\right) \\
C\left(\omega_{2}\right)= & \frac{1}{2}\left[\left(q_{22} \omega_{2}^{2}-q_{20}\right)^{2}+\left(q_{23} \omega_{2}^{2}-q_{21} \omega_{2}\right)^{2}-\left(q_{34} \omega_{2}^{4}-q_{32} \omega_{2}^{2}\right)^{2}\right. \\
& \left.-\left(\omega_{2}^{5}+q_{23} \omega_{2}^{2}-q_{31} \omega_{2}\right)^{2}-\left(q_{10}-q_{12} \omega_{2}^{2}\right)^{2}-\left(q_{11} \omega_{2}-q_{13} \omega_{2}^{3}\right)^{2}\right] \\
E_{1}\left(\omega_{2}\right)= & q_{34} \omega_{2}^{4}-q_{32} \omega_{2}^{2}+q_{30}+\left(q_{10}-q_{12} \omega_{2}^{2}\right) \cos \left(\omega_{2} \tau_{1}^{*}\right) \\
& +\left(q_{11} \omega_{2}-q_{13} \omega_{2}^{3}\right) \sin \left(\omega_{2} \tau_{1}^{*}\right), \\
E_{2}\left(\omega_{2}\right)= & \omega_{2}^{5}+q_{33} \omega_{2}^{3}-q_{31} \omega_{2}-\left(q_{11} \omega_{2}-q_{13} \omega_{2}^{3}\right) \cos \left(\omega_{2} \tau_{1}^{*}\right) \\
& +\left(q_{11}-q_{12} \omega_{2}^{2}\right) \sin \left(\omega_{2} \tau_{1}^{*}\right) .
\end{aligned}
$$

Proposition 7 Suppose conditions (40) and $q_{20}+q_{30}-q_{10}<0$ hold. Consider $\tau_{1}^{*} \in\left[0, \tau_{10}\right)$, where $\tau_{10}$ is given by $(53)$. Let $\omega_{20}$ be a positive solution of

$$
F\left(\omega_{2}\right):=A\left(\omega_{2}\right) \cos \left(\omega_{2} \tau_{1}^{*}\right)+B\left(\omega_{2}\right) \sin \left(\omega_{2} \tau_{1}^{*}\right)-C\left(\omega_{2}\right)=0
$$

and $\tau_{20}$ is given by: 


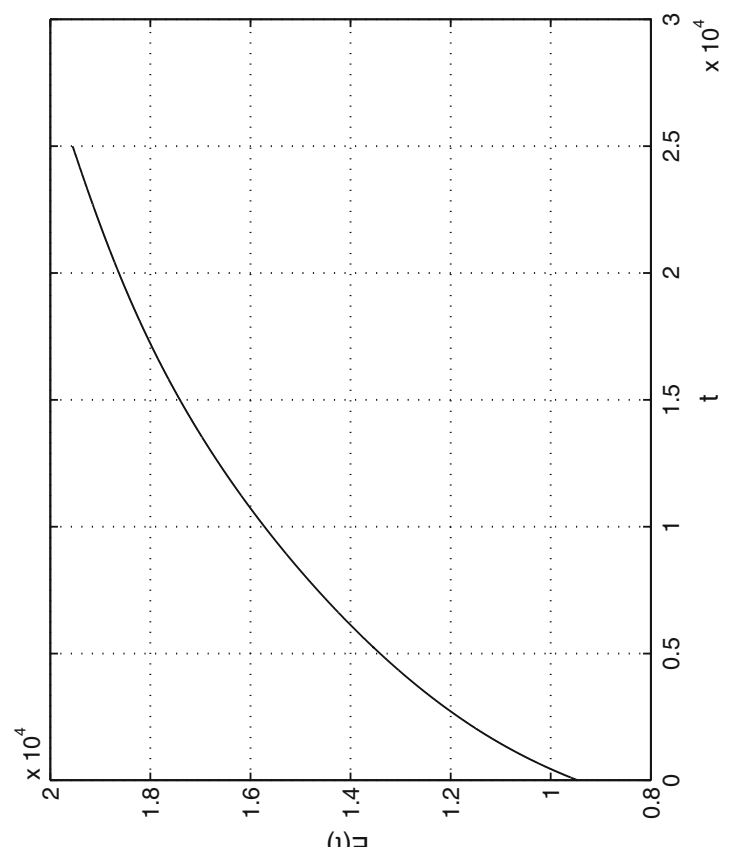

(1) $\exists$

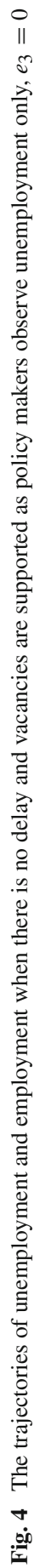




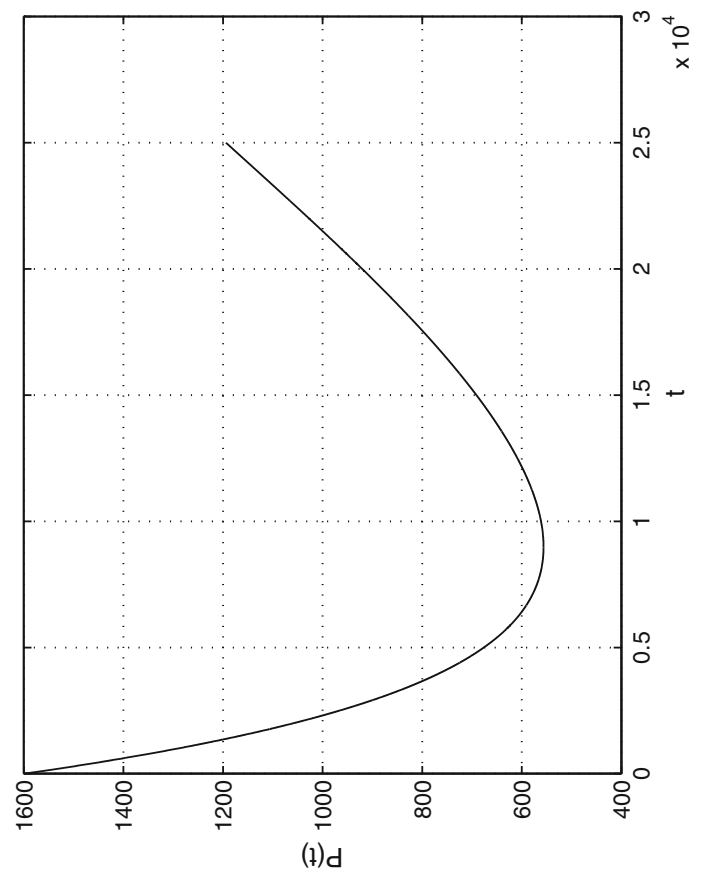

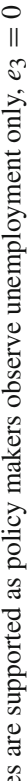

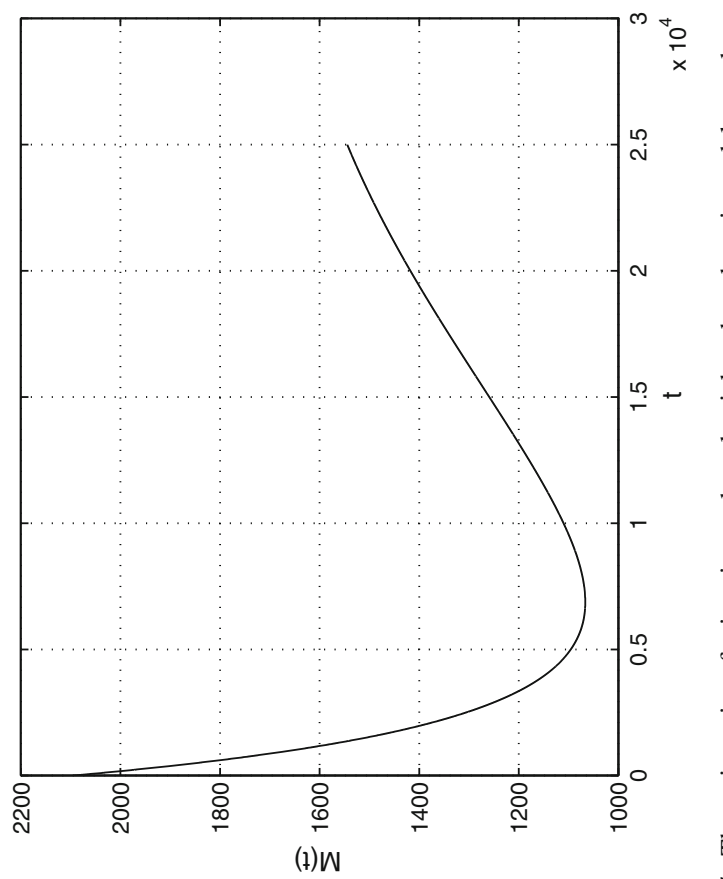

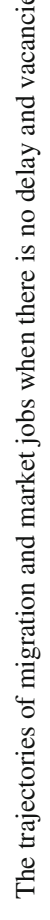

我 


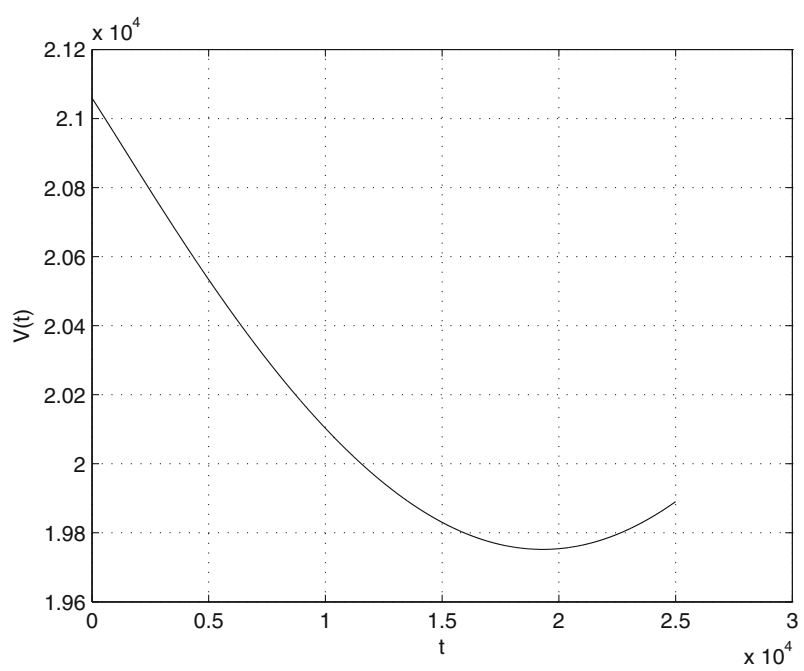

Fig. 6 The trajectories of vacancies created through government intervention when there is no delay and vacancies are supported as policy makers observe unemployment only, $e_{3}=0$

$$
\begin{aligned}
\tau_{20}= & \frac{1}{\omega_{20}}\left[\arccos \left(\frac{E_{1}\left(\omega_{20}\right)\left(q_{22} \omega_{20}^{2}-q_{20}\right)-E_{2}\left(\omega_{20}\right)\left(q_{23} \omega_{20}^{2}-q_{21} \omega_{21}\right)}{\left(q_{22} \omega_{20}^{2}-q_{20}\right)^{2}+\left(q_{23} \omega_{20}^{2}-q_{21} \omega_{21}\right)^{2}}\right)+2 n \pi\right], \\
& n=0,1,2, \ldots
\end{aligned}
$$

The value $\tau_{2}=\tau_{20}$ is a Hopf bifurcation. For $\tau_{2} \in\left[0, \tau_{20}\right)$ the equilibrium point $E_{0}$ is locally asymptotically stable. For $\tau_{2}>\tau_{20}$ the equilibrium point $E_{0}$ is unstable. If $\tau_{2}=\tau_{20}$ the given system has a limit cycle in the neighbourhood of the equilibrium point.

In what follows we study the case $k_{1}\left(d_{1}, s\right)=d_{1} e^{-d_{1} s}$ and $k_{2}\left(d_{1}, s\right)=d_{2} e^{-d_{2} s}$, with $d_{1}>0, d_{2}>0$.

Equation (37) becomes:

$$
Q_{3}(\lambda)+Q_{1}(\lambda) \frac{d_{1}}{\lambda+d_{1}}+Q_{2}(\lambda) \frac{d_{2}}{\lambda+d_{2}}=0
$$

where $Q_{1}, Q_{2}, Q_{3}$ are given by (38).

From (60) with (38) we obtain the equation:

$$
\lambda^{7}+B_{6} \lambda^{6}+B_{5} \lambda^{5}+b_{4} \lambda^{4}+B_{3} \lambda^{3}+B_{2} \lambda^{2}+B_{1} \lambda+B_{0}=0,
$$



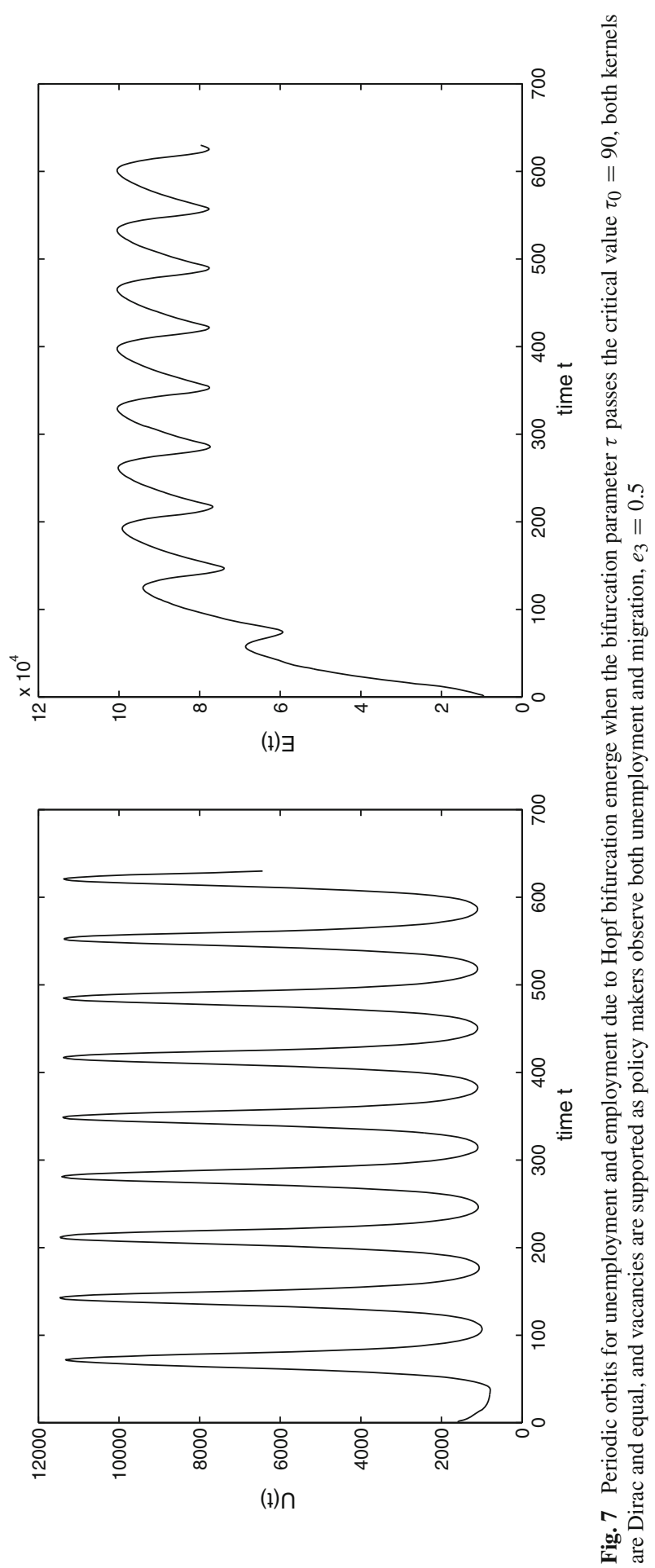

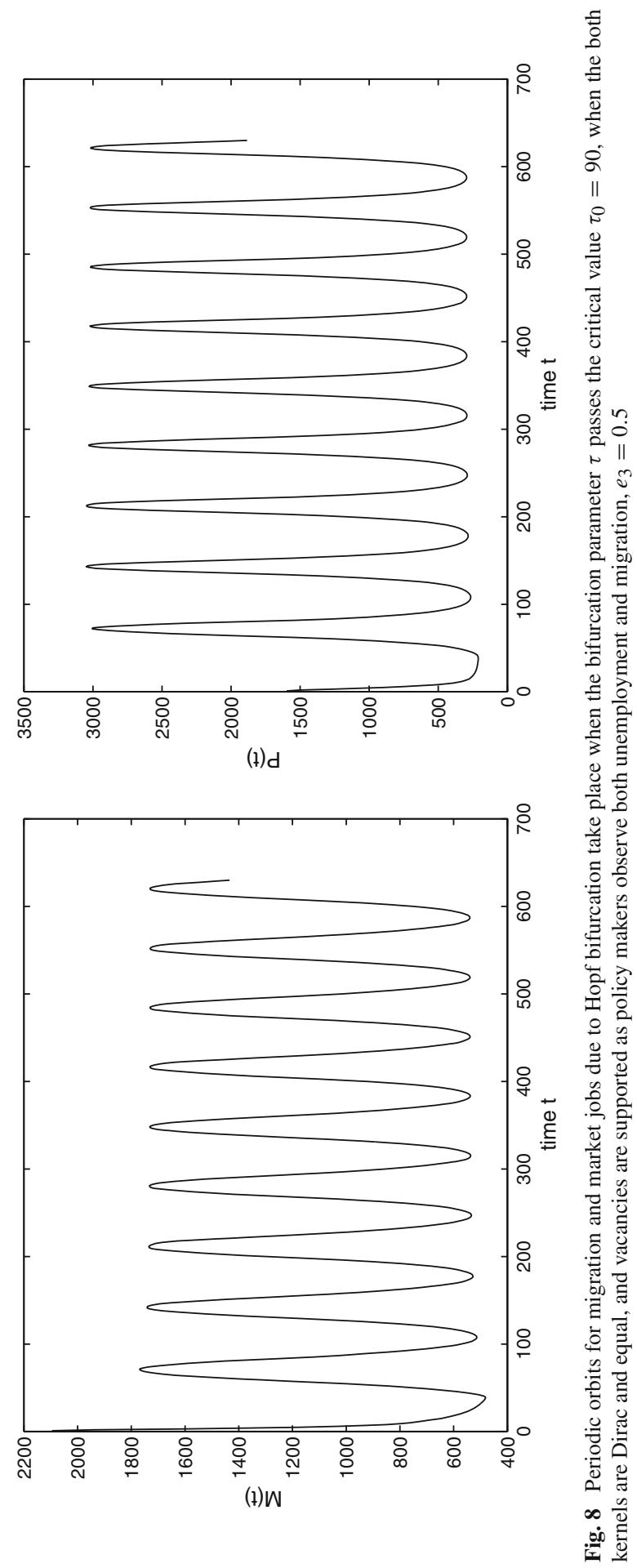


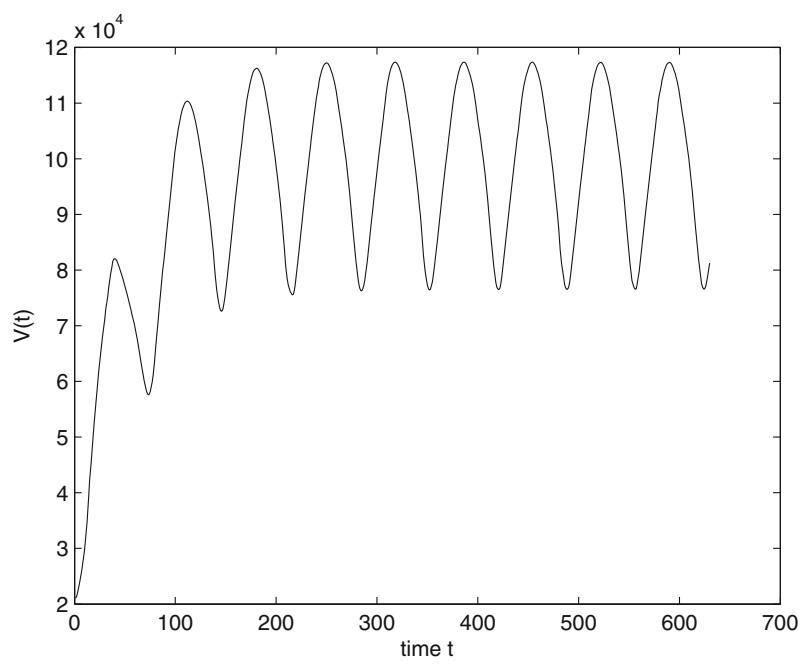

Fig. 9 Periodic orbit for vacancies through government intervention due to Hopf bifurcation take place when the bifurcation parameter $\tau$ passes the critical value $\tau_{0}=90$, both kernels are Dirac and equal, and vacancies are supported as policy makers observe both unemployment and migration, $e_{3}=0.5$

where

$$
\begin{aligned}
& B_{6}=q_{34}+d_{1}+d_{2}, B_{5}=q_{33}+\left(d_{1}+d_{2}\right) q_{34}+d_{1} d_{2}, \\
& B_{4}=q_{34}+q_{32}+\left(d_{1}+d_{2}\right) q_{33}+d_{1} q_{13}+d_{2} q_{23}, \\
& B_{3}=q_{31}+q_{32}\left(d_{1}+d_{2}\right)+d_{1} d_{2}\left(q_{33}+q_{23}+q_{13}\right)+d_{1} q_{12}+d_{2} q_{22}, \\
& B_{2}=\left(d_{1}+d_{2}\right) q_{31}+d_{1} d_{2}\left(q_{12}+q_{22}+q_{32}\right)+d_{2} q_{21}+q_{11} d_{11}, \\
& B_{1}=\left(d_{1}+d_{2}\right) q_{30}+d_{1} d_{2}\left(q_{32}+q_{21}+q_{11}\right)+d_{1} q_{10}+d_{2} q_{20}, \\
& B_{0}=d_{1} d_{2}\left(q_{30}+q_{20}+q_{10}\right) .
\end{aligned}
$$

We consider:

$$
\begin{aligned}
D_{1} & =B_{1}, D_{2}=B_{1} B_{2}-B_{0} B_{3}, D_{3}=\left|\begin{array}{ccc}
B_{1} & B_{0} & 0 \\
B_{3} & B_{2} & B_{1} \\
B_{5} & B_{4} & B_{3}
\end{array}\right| \\
D_{4} & =\left|\begin{array}{cccc}
B_{1} & B_{0} & 0 & 0 \\
B_{3} & B_{2} & B_{1} & B_{0} \\
B_{5} & B_{4} & B_{3} & B_{2} \\
1 & B_{6} & B_{5} & B_{4}
\end{array}\right|, D_{5}=\left|\begin{array}{ccccc}
B_{1} & B_{0} & 0 & 0 & 0 \\
B_{3} & B_{2} & B_{1} & B_{0} & 0 \\
B_{5} & B_{4} & B_{3} & B_{2} & B_{1} \\
1 & B_{6} & B_{5} & B_{4} & B_{3} \\
0 & 0 & 1 & B_{6} & B_{5}
\end{array}\right|, \\
D_{6} & =\left|\begin{array}{cccccc}
B_{1} & B_{0} & 0 & 0 & 0 & 0 \\
B_{3} & B_{2} & B_{1} & B_{0} & 0 & 0 \\
B_{5} & B_{4} & B_{3} & B_{2} & B_{1} & B_{0} \\
1 & B_{6} & B_{5} & B_{4} & B_{3} & B_{2} \\
0 & 0 & 1 & B_{6} & B_{5} & B_{4} \\
0 & 0 & 0 & 0 & 1 & B_{0}
\end{array}\right| .
\end{aligned}
$$




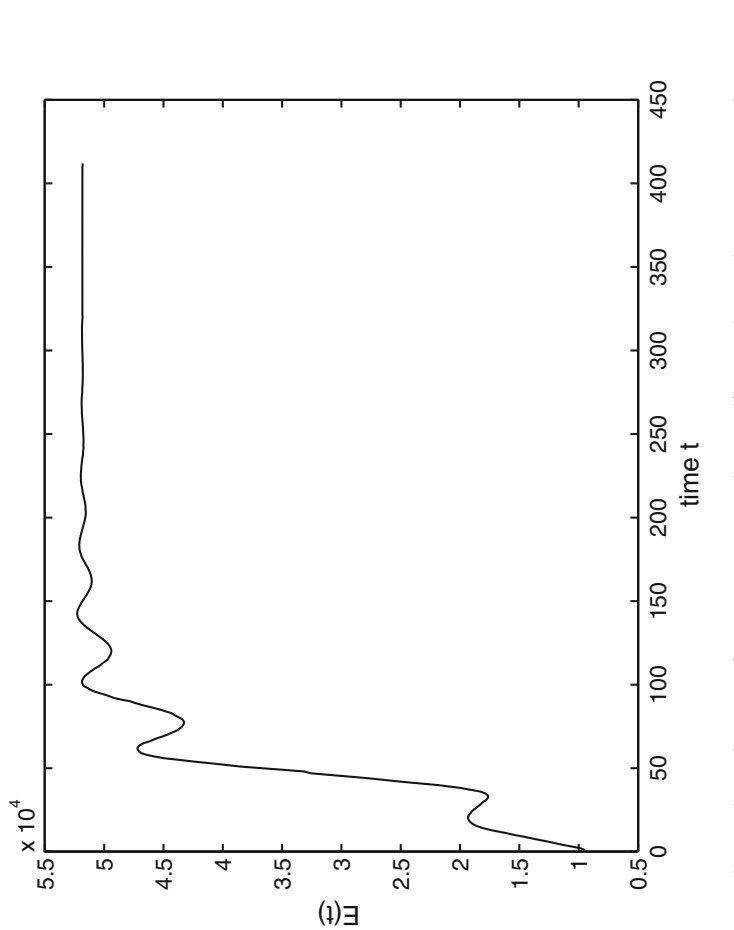

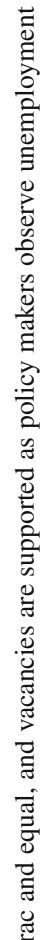

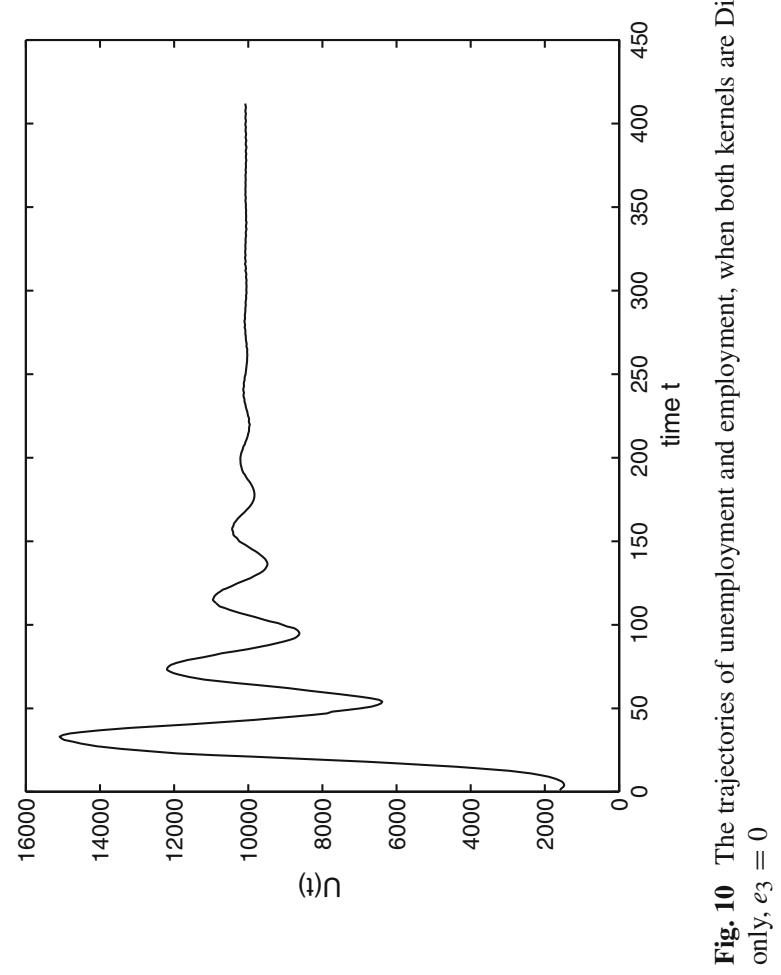




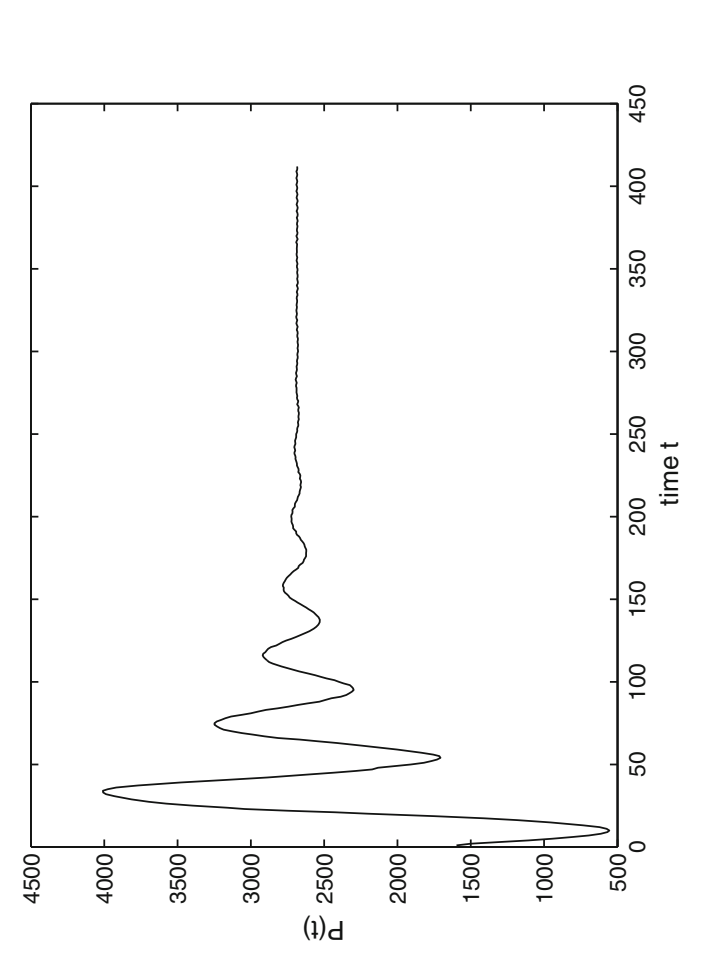

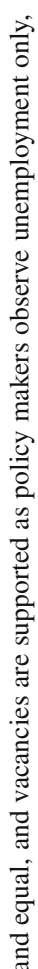

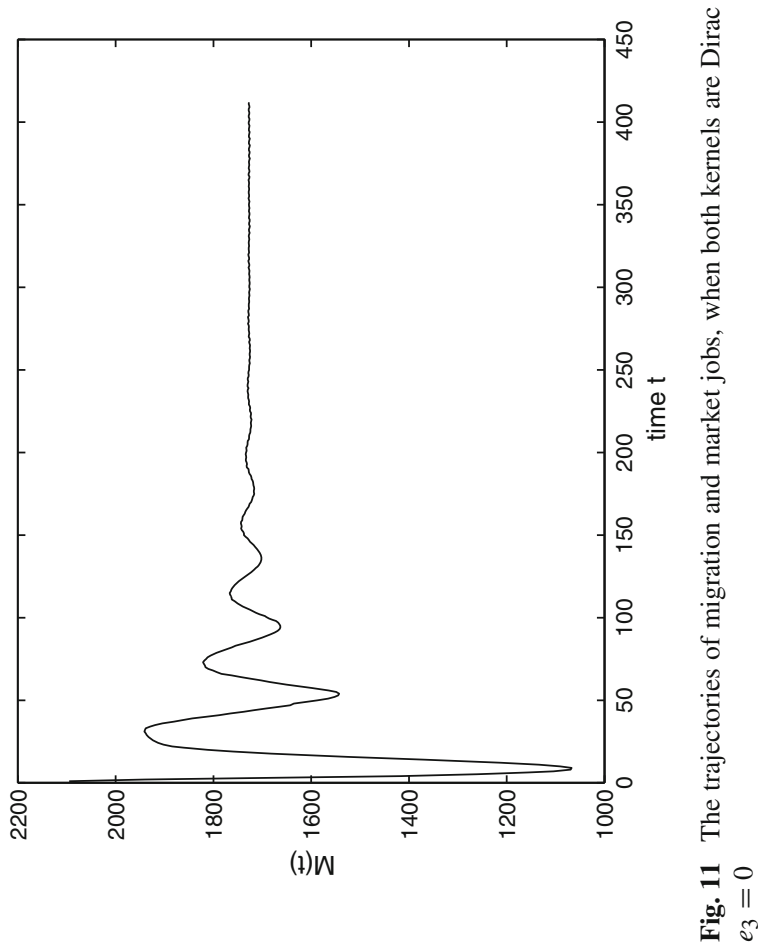




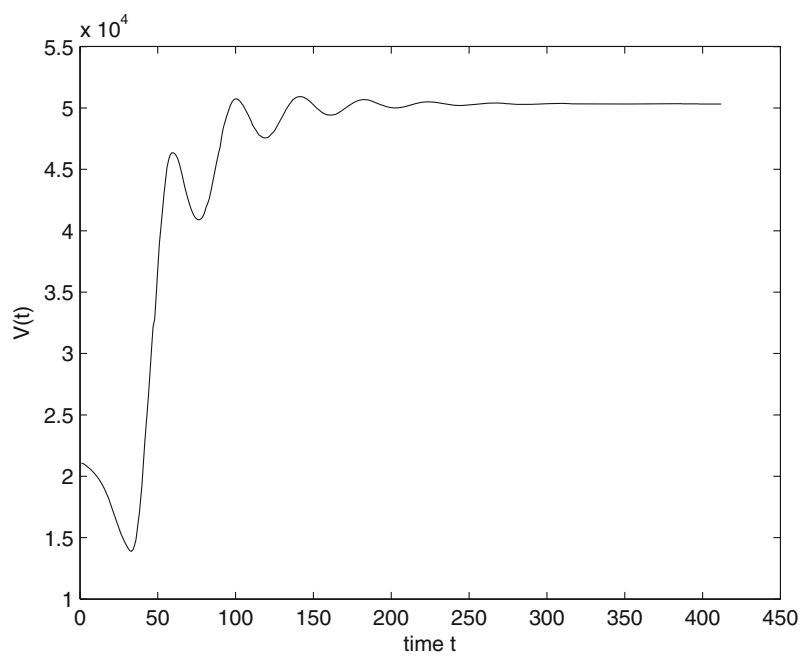

Fig. 12 The trajectory of vacancies through government intervention, when both kernels are Dirac and equal, and vacancies are supported as policy makers observe unemployment only, $e_{3}=0$

Proposition 8 If conditions $D_{1}>0, D_{2}>0, D_{3}>0, D_{4}>0, D_{5}>0, D_{6}>0$ hold for any $d_{i}>0, i=1,2$, the equilibrium point $E_{0}$ of system (1)-(5) is locally asymptotically stable.

\section{Numerical Simulations and Discussions}

In the next step we will simulate the outcome of the system choosing parameters informed by the economic literature, and specific delays. We present results where policy responds to both unemployment and migration, illustrating the behaviour of the system introduced theoretically. We further compare an outcome of our system in which we let government disregard migrant numbers in its policy reaction, with the more comprehensive approach in which policy responds to unemployment and migration alike.

For the numerical simulation we consider the following data: $a_{1}=500, a_{2}=$ $0.00004, a_{3}=0.04, a_{4}=0.004, a_{5}=0.1, m_{1}=300, m_{2}=0.00002, m_{3}=0.05$, $b_{1}=0.006, c_{1}=0.08, c_{2}=0.3, e_{1}=0.04, e_{2}=0.008$.

First, we simulate the situation where there are no delays in the system, and both migration and unemployment are factored into the promotion of vacancies.

If there is no delay, the conditions from Proposition 2 hold. Therefore the equilibrium point is locally asymptotically stable. In Figs. 1, 2 and 3, we can visualise the evolution of unemployment, employment, migration, market jobs and vacancies created through government intervention.

Next, we consider a case where vacancies are supported on the observation by policy makers of unemployment alone, disregarding the fact that migrants can take up some newly created jobs. Under this scenario, with no delays in the system and 


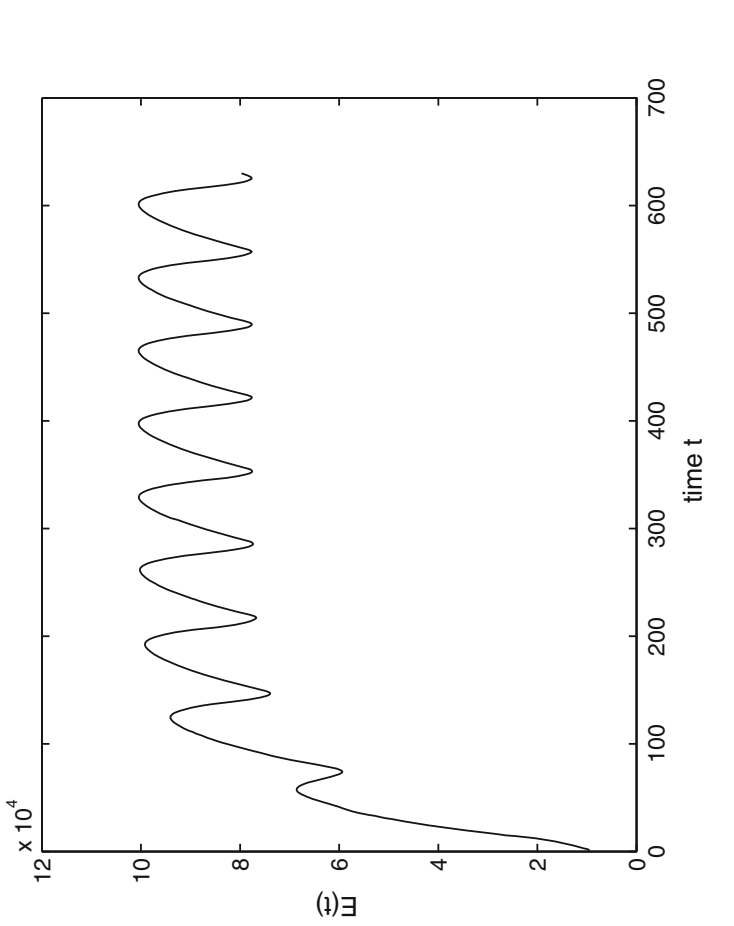

ฏ

告

吾

苟

(2)

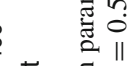

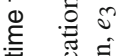

总

() $\exists$

굴

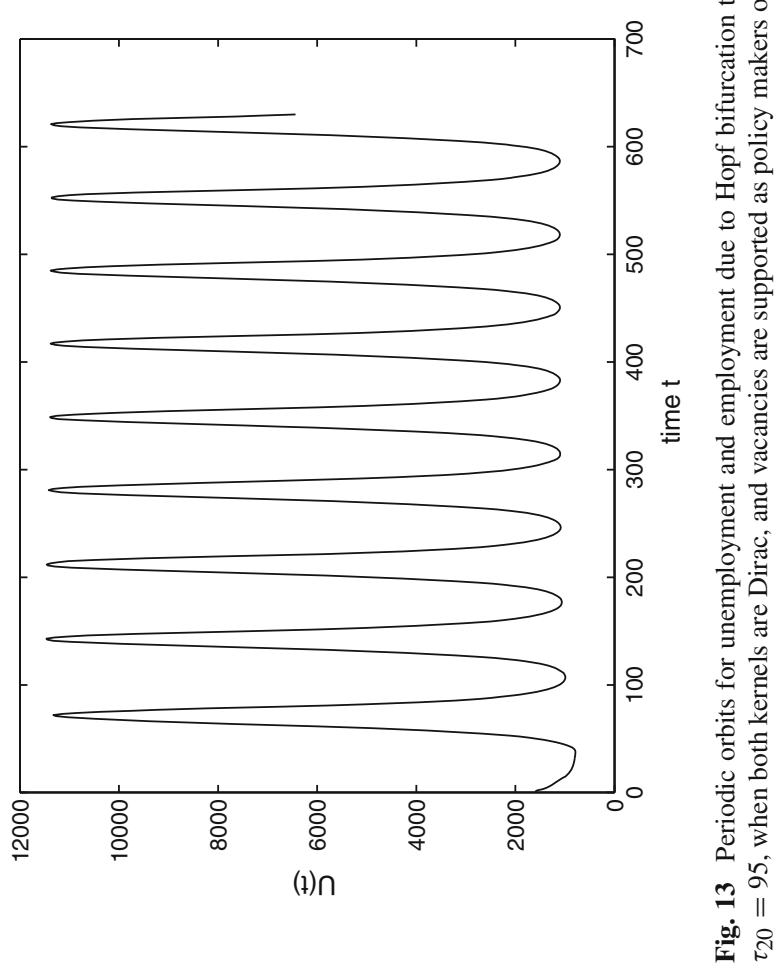




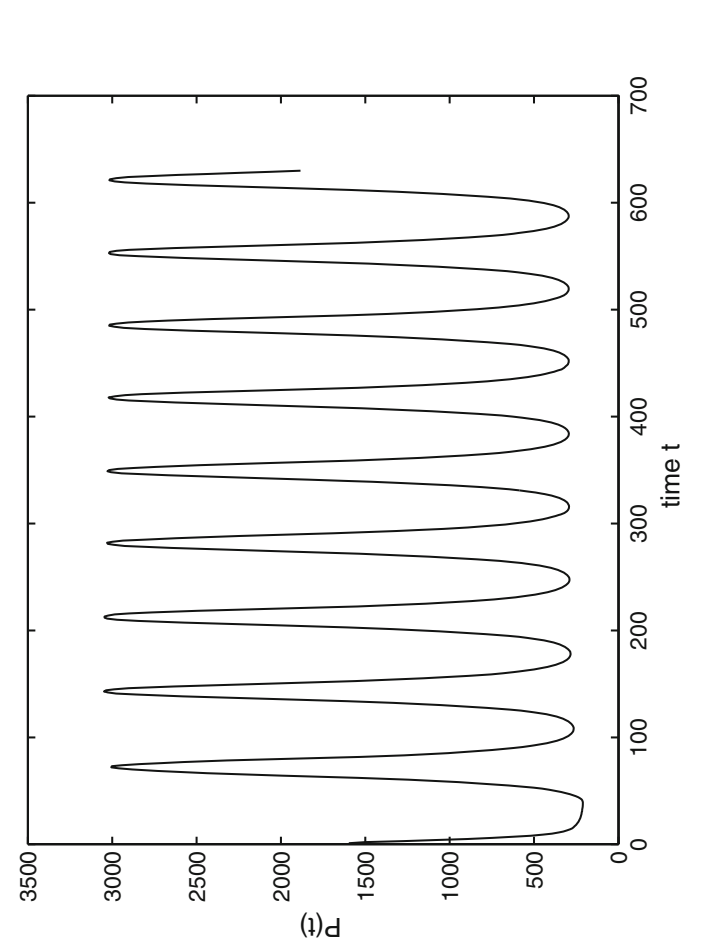

ผ่

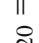

긴

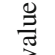

氖

Е

w

(1)

:

.

氖.

吾.

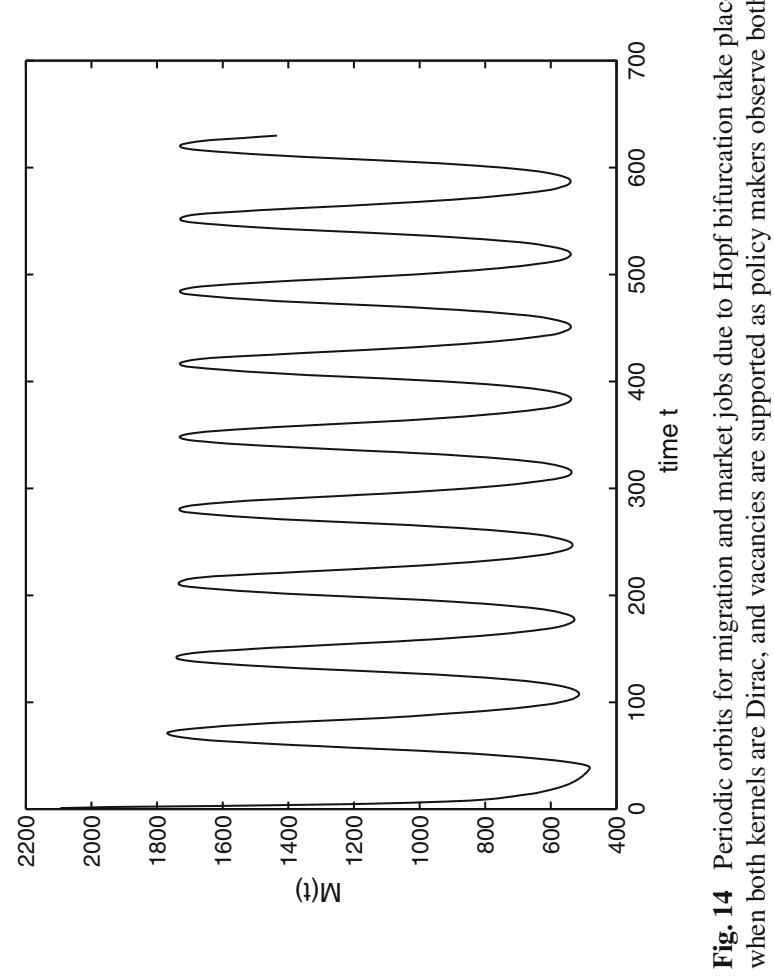




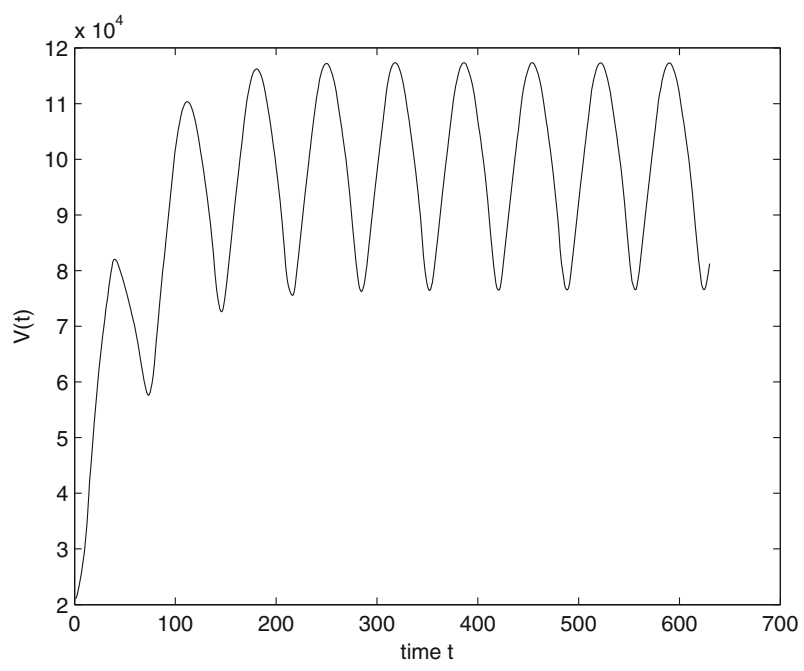

Fig. 15 Periodic orbit for vacancies through government intervention due to Hopf bifurcation take place when $\tau_{10}=90$ and the bifurcation parameter $\tau_{2}$ passes the critical value $\tau_{20}=95$, when both kernels are Dirac, and vacancies are supported as policy makers observe both unemployment and migration, $e_{3}=0.5$

$e_{3}=0$, we present the orbits of unemployment, employment, migration, market jobs and vacancies through government intervention in Figs. 4, 5 and 6.

We would like to make a few interesting observations with respect to the simulated outcomes (over the same time framework), in particular with respect to unemployment. While unemployment declines for a little while in both scenarios, we have a much smaller initial decline in unemployment where migration is ignored in Fig. 4, and a much stronger rise in unemployment over time. The better outcome in unemployment levels in Fig. 1 is sustained through the ongoing action by policy makers where they take migration into account, whereas the government support for new vacancies is gradually reduced in the alternative scenario of Fig. 6. The market addition of a larger number of jobs where government ignores migration in Fig. 5 partly compensates for the lower number of vacancies supported through intervention. Yet, this is not enough to bring unemployment down to the level observed where the government considered migration in its policy formulation (see Fig. 1 vs. Fig. 4). Hence, we can conclude that where unemployment is the main target of policy and migration is part of the system, unemployment levels are much higher should policy ignore the inflow of migrant workers searching jobs along with natives.

We shall concentrate next on the simulation of our model where government responds, with a delay, to both unemployment and migration. If both kernels are Dirac $k_{i}\left(\tau_{i}, s\right)=\delta\left(s-\tau_{i}\right)$ with $\tau_{1}=\tau_{2}=\tau$, according to Theorem 3, there exists a Hopf bifurcation for $\tau_{0}=90$ and a limit cycle. In Fig. 7, 8 and 9 the orbits of unemployment, employment, migration, market jobs and vacancies through government intervention are displayed.

In the same scenario with both kernels Dirac $k_{i}\left(\tau_{i}, s\right)=\delta\left(s-\tau_{i}\right), \tau_{1}=\tau_{2}=\tau$, we simulate the case when vacancies are supported on the observation by policy makers of unemployment alone, $e_{3}=0$. In Figs. 10, 11 and 12 we have the orbits of 


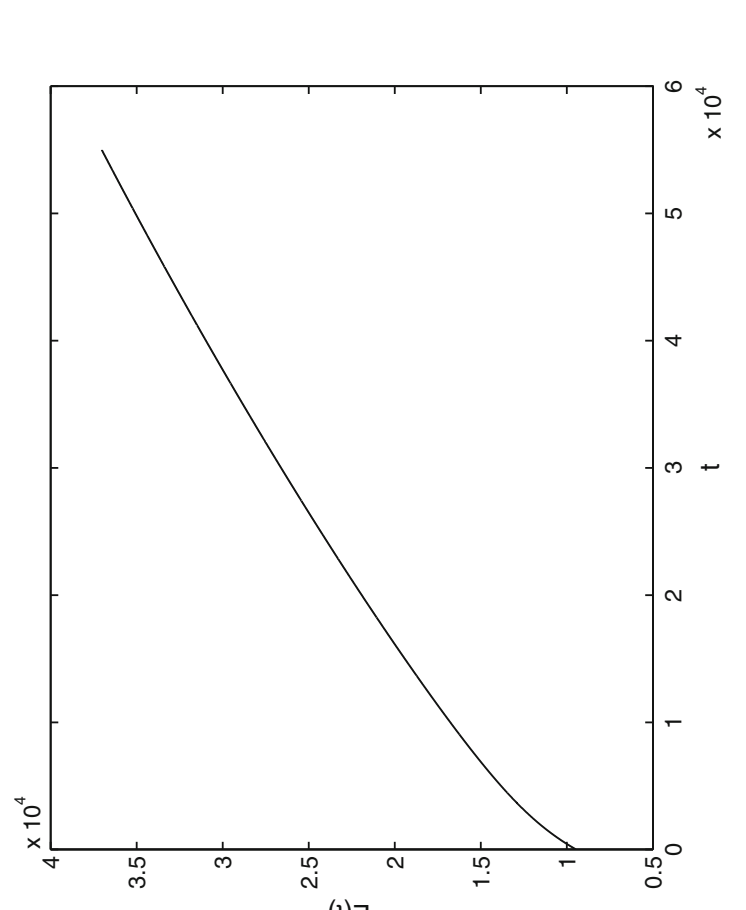

列

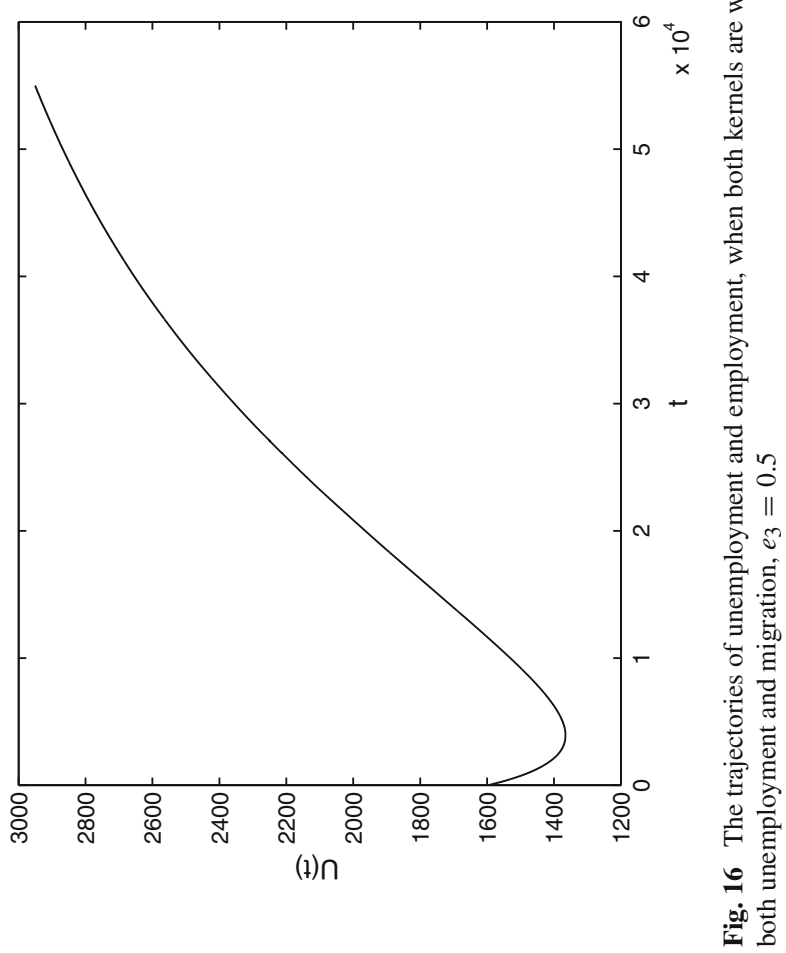




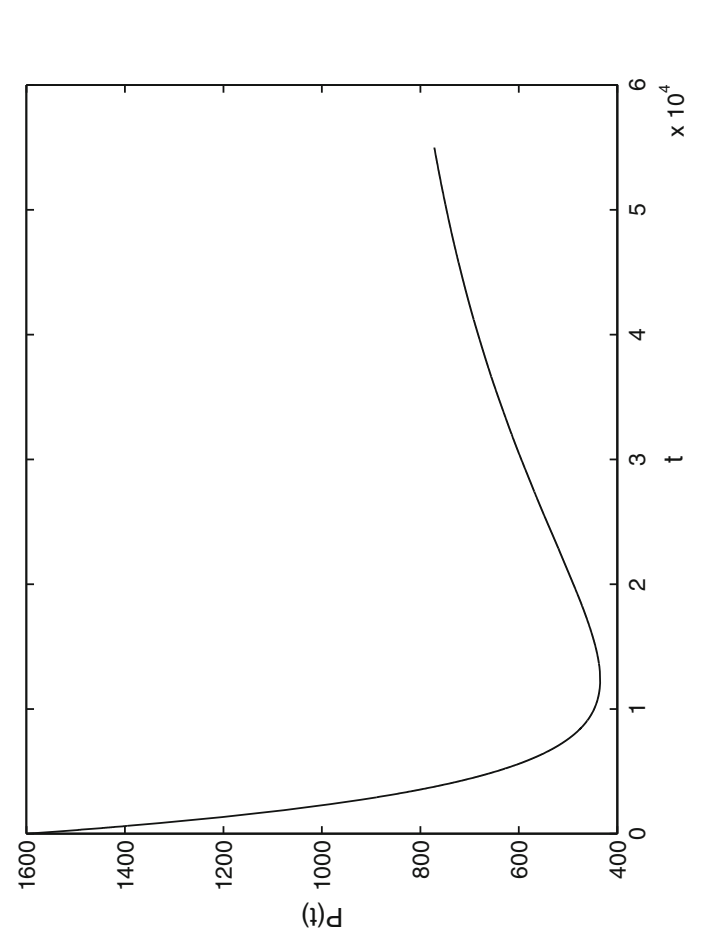

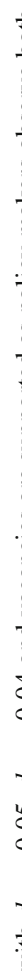

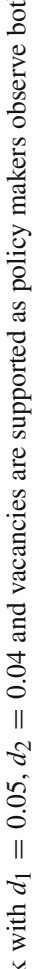

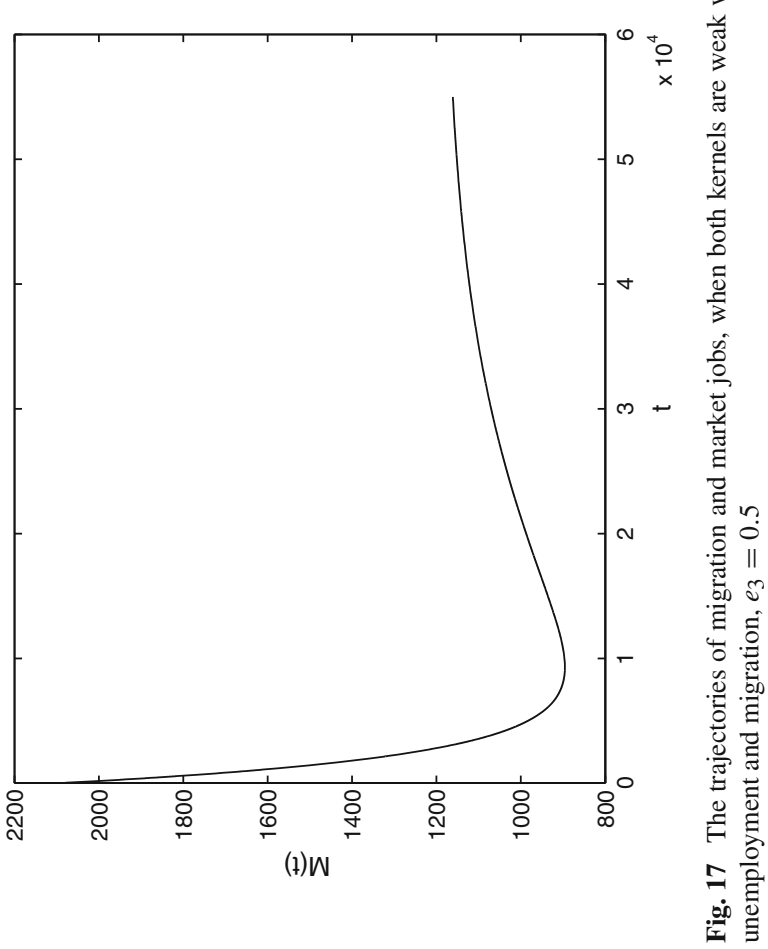




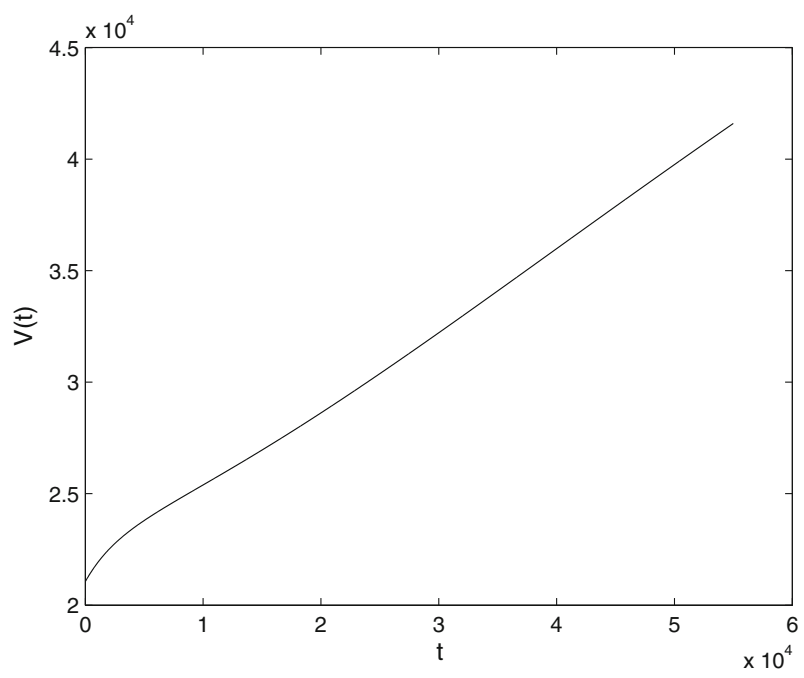

Fig. 18 The trajectories of vacancies through government intervention, when both kernels are weak with $d_{1}=0.05, d_{2}=0.04$ and vacancies are supported as policy makers observe both unemployment and migration, $e_{3}=0.5$

unemployment, employment, migration, market jobs and vacancies through government intervention. They converge to the asymptotically stable equilibrium point. We emphasise that having ignored migration, policy intervention this time around results in a stable outcome.

We find that if both kernels are Dirac $k_{1}\left(\tau_{1}, s\right)=\delta\left(s-\tau_{1}\right), k_{2}\left(\tau_{2}, s\right)=\delta\left(s-\tau_{2}\right)$, due to Proposition 7, then there exists a Hopf bifurcation for $\tau_{20}=95$, when $\tau_{10}=90$. If $\tau_{2}=\tau_{20}$, there exists a limit cycle. In Figs. 13, 14 and 15 we can notice that the orbits of unemployment, employment, migration, market jobs and vacancies through government intervention all oscillate.

Finally, to further illustrate the system behaviour under an alternative specification we simulate the system with weak kernels in policy responses to both migration and unemployment. If both kernels are weak $k_{1}\left(d_{1}, s\right)=d_{1} e^{-d_{1} s}, k_{2}\left(d_{2}, s\right)=d_{2} e^{-d_{2} s}$, with $d_{1}=0.05, d_{2}=0.04$, the equilibrium point is locally asymptotically stable due to Proposition 8. Figures 16, 17 and 18 show the orbits of unemployment, employment, migration, market jobs and vacancies through government intervention.

We notice that the numerical simulations in Figs. 1, 2, 3, 4, 5, 6, 7, 8, 9, 10, 11, 12 , 13,14 and 15 verify the theoretical findings of Sect. 4.

\section{Conclusions}

The paper developed and analysed a model for unemployment reduction where ongoing migration takes place. This is described by a nonlinear differential system with distributed time delay. At any time $t$, we take into account five variables: the number of unemployed individuals, the number of employed individuals, the number of new immigrants, the number of total jobs on the market, and the number of vacancies newly created through government intervention. 
For the unique positive equilibrium point we study the local asymptotic stability according to distributed time delays. In absence of delay the equilibrium point is locally asymptotically stable under some conditions of the parameters.

We have tested the significance of taking migration into account when formulating policy to address unemployment. We thus observed the evolution of unemployment, employment, migration, market jobs and government supported vacancies with two alternative policy approaches. In a first instance we have simulated the system in the case where policy aims to reduce unemployment by observing both past values of unemployment and migration. In the second instance policy supports jobs creation by taking into account past unemployment alone. Where migrants are taken into account by the policy, a more significant drop is registered in initial levels of unemployment and a lower level of unemployment results over time. In the second scenario where government ignores migration, unemployment quickly returns to a relatively high value after a small dip.

In the case of Dirac kernels it is proved that there is a Hopf bifurcation and the stable equilibrium becomes unstable as delay crosses some critical values. Yet, where the kernels are equal and government ignores migration, policy intervention results in a stable outcome.

Where both kernels are weak, under some conditions of parameters, the equilibrium point is locally asymptotically stable.

Our theoretical findings are tested in a set of simulations including locally asymptotic stable outcomes and limit cycles.

For the numerical simulations we used Maple 17 and Matlab and the figures obtained verify the theoretical statements.

For the uniform distribution and the strong kernel, a similar analysis will be carried out in a further paper. Also, the direction of the Hopf bifurcation can be analysed. As in Mircea et al. (2011) the stochastic approach will be taken into consideration.

Acknowledgements We thank Professor Dumitru Opris for useful conversations on the topics of this paper. Thanks go as well to the editor and an anonymous reviewer for their essential comments.

Open Access This article is distributed under the terms of the Creative Commons Attribution 4.0 International License (http://creativecommons.org/licenses/by/4.0/), which permits unrestricted use, distribution, and reproduction in any medium, provided you give appropriate credit to the original author(s) and the source, provide a link to the Creative Commons license, and indicate if changes were made.

\section{Appendix 1: The Proof of Lemma}

From (1)-(3) we obtain:

$$
\frac{d}{d t}(U(t)+M(t)+E(t))=a_{1}+m_{1}-a_{3} U(t)-m_{3} M(t)-b_{1} E(t)
$$

which leads to

$$
\frac{d}{d t}(U(t)+M(t)+E(t)) \leq a_{1}+m_{1}-\delta_{m}(U(t)+M(t)+E(t))
$$


Taking the supremum limit, we obtain:

$$
\limsup _{t \rightarrow \infty}[U(t)+M(t)+E(t)] \leq \frac{a_{1}+m_{1}}{\delta_{m}}
$$

From (4), we have:

$$
\frac{d V(t)}{d t} \leq\left(e_{1}+e_{3}\right) U(t)-e_{2} V(t) .
$$

This implies

$$
\limsup _{t \rightarrow \infty} V(t) \leq \frac{\left(e_{1}+e_{3}\right)\left(a_{1}+m_{1}\right)}{\delta e_{2}}
$$

From (5), we have:

$$
\limsup _{t \rightarrow \infty} P(t) \leq \frac{c_{1}\left(a_{1}+m_{1}\right)}{\delta c_{2}}
$$

that proves our lemma.

\section{Appendix 2: The Proof of Proposition 1}

Let the function:

$$
f(x)=\gamma_{3} x^{3}+\gamma_{2} x^{2}+\gamma_{1} x+\gamma_{0} .
$$

From (27) we have $f(0)=\gamma_{0}<0$ and $f^{\prime}(x)=0$ has the roots $x_{M}, x_{m}$ with $x_{M}<0, x_{m}>0$. Therefore, for $x \in\left[0, x_{m}\right], f^{\prime}(x)<0$ and for $x \in\left[x_{m}, \infty\right)$, $f^{\prime}(x)>0$. Thus, Eq. (26) has one root $x_{10} \in\left[x_{m}, \infty\right)$. Moreover, it can be proved that $x_{10} \in\left(\frac{\alpha_{01}}{\alpha_{11}}, \frac{\beta_{01}}{\beta_{11}}\right)$.

\section{Appendix 3: The Proof of Proposition 2}

If $\mathscr{E}=0$, the characteristic Eq. (37) becomes:

$$
\begin{aligned}
& \lambda^{5}+q_{34} \lambda^{4}+\left(q_{13}+q_{23}+q_{33}\right) \lambda^{3}+\left(q_{12}+q_{22}+q_{32}\right) \lambda^{2} \\
& \quad+\left(q_{11}+q_{21}+q_{31}\right) \lambda+q_{10}+q_{20}+q_{30}=0 .
\end{aligned}
$$

From (39), we have $q_{34}>0, q_{13}+q_{23}+q_{33}>0, q_{12}+q_{22}+q_{32}>0, q_{11}+q_{21}+$ $q_{31}>0, q_{10}+q_{20}+q_{30}>0$ and an algebraic manipulation yields to the inequalities (40). Therefore, by using the Routh-Hurwitz criterion, we can say that all the roots of equation (68) are either negative or have a negative real part. Thus, the proposition is proved. 


\section{Appendix 4: The Proof of Theorem 1}

Eq. (41) does not have the root $\lambda=0$, because $q_{10}+q_{20}+q_{30}>0$. Assume that for some expectation $\mathscr{E}_{1}\left(d_{1}\right), \lambda=i \omega(\omega>0)$ is a root of (41). Substituting $\lambda=i \omega$ into (41) and separating the real and imaginary parts, we have:

$$
\begin{aligned}
& \left(q_{10}+q_{20}-\left(q_{12}+q_{22}\right) \omega^{2}\right) \int_{0}^{\infty} k_{1}\left(d_{1}, s\right) \cos (\omega s) d s \\
& \quad+\left(\left(q_{11}+q_{21}\right) \omega-\left(q_{13}+q_{23}\right) \omega^{3}\right) \int_{0}^{\infty} k_{1}\left(d_{1}, s\right) \sin (\omega s) d s \\
& =q_{32} \omega^{2}-q_{30}-q_{34} \omega^{4} . \\
& \left(\left(q_{11}+q_{21}\right) \omega-\left(q_{13}+q_{23}\right) \omega^{3}\right) \int_{0}^{\infty} k_{1}\left(d_{1}, s\right) \cos (\omega s) d s \\
& \quad-\left(\left(q_{10}+q_{21}-\left(q_{12}+q_{22}\right) \omega^{2}\right) \int_{0}^{\infty} k\left(d_{1}, s\right) \sin (\omega s) d s\right. \\
& =\omega^{5}+q_{23} \omega^{3}-q_{21} \omega
\end{aligned}
$$

Adding sidewise after squaring the left and right sides of (69) and (70), we can obtain the equations:

$$
\omega^{10}+r_{8} \omega^{8}+r_{6} \omega^{6}+r_{4} \omega^{4}+r_{2} \omega^{2}+r_{0}=0,
$$

where

$$
\begin{aligned}
& r_{8}=q_{34}^{2}+2 q_{33}, r_{6}=q_{33}^{2}-\left(q_{13}+q_{23}\right)^{2}-2 q_{31}-2 q_{32} q_{34}, \\
& r_{0}=q_{30}^{2}-\left(q_{10}+q_{20}\right)^{2}, \\
& r_{4}=q_{32}^{2}-\left(q_{12}+q_{22}\right)^{2}+2 q_{34} q_{30}-2 q_{31} q_{33}+2\left(q_{11}+q_{21}\right)\left(q_{13}+q_{23}\right), \\
& r_{2}=q_{31}^{2}-\left(q_{11}+q_{21}\right)^{2}-2 q_{32} q_{30}+2\left(q_{10}+q_{20}\right)\left(q_{12}+q_{22}\right) .
\end{aligned}
$$

Because $r_{0}=q_{30}^{2}-\left(q_{10}+q_{20}\right)^{2}<0$, then Eq. (71) has a positive root $\omega_{0}$ and $\omega \in\left(0, \omega_{0}\right]$.

\section{Appendix 5: The Proof of Theorem 3}

If $\tau=0$, the characteristic equation (42) has the roots with negative real part. If $\tau=\tau_{0}$, Eq. (42) has a pair of conjugate purely imaginary roots $\pm i \omega_{0}$. If $\tau \in\left[0, \tau_{0}\right)$, Eq. (42) has roots with negative real part. Thus, if $\tau \in\left[0, \tau_{0}\right)$, the equilibrium point $E_{0}$ is locally asymptotically stable.

Let denote the root of (45) by $\lambda(\tau)=\mu(\tau)+i \omega(\tau)$, then we have:

$$
\mu\left(\tau_{0}\right)=0, \omega\left(\tau_{0}\right)=\omega_{0} .
$$

If the transversal condition $\mu\left(\tau_{0}\right)=\left.\frac{d}{d \tau}(\operatorname{Re}(\lambda(\tau)))\right|_{\tau=\tau_{0}} \neq 0$ is satisfied, then the Hopf bifurcation occurs in the system (1)-(5) when $\tau=\tau_{0}$.

By straight calculus we obtain (45). 
Thus if condition 2 of the theorem holds, the Hopf bifurcation occurs as the transversal condition holds.

\section{References}

Angrist, J. D., \& Kugler, A. D. (2003). Protective or counter-productive? Labour market institutions and the effect of immigration on EU natives. The Economic Journal, 113(488), F302-F331.

Bauer, T., \& Zimmermann, K. F. (1999). Assessment of possible migration pressure and its labour market impact following EU enlargement to central and eastern Europe. A Study for the Department for Education and Employment, London.

Bernard, S., Belair, J., \& Mackey, M. C. (2001). Sufficient conditions for stability of linear differential equations with distributed delay. Discrete and Continuous Dynamical Systems, 1, 233-256.

Blanchflower, D. G., \& Shadforth, C. (2009). Fear, unemployment and migration. The Economic Journal, 119(535), F136-F182.

Camacho, C. (2013). Migration modelling in the new economic geography. Mathematical Social Sciences, $66,233-244$.

Damette, O., \& Fromentin, V. (2013). Migration and labour markets in OECD countries: A panel cointegration approach. Applied Economics, 45(16), 2295-2304.

Dustmann, Ch., \& Frattini, T. (2013). The fiscal effects of immigration to the UK, CReAM Discussion Paper Series 1322, Centre for Research and Analysis of Migration (CReAM), Department of Economics: University College London.

Facchini, G., \& Mayda, A. M. (2008). From individual attitudes towards migrants to migration policy outcomes: Theory and evidence. Economic Policy, 23(56), 651-713.

Hatton, T. J. (2014). The Slump and immigration policy in Europe. CReAM Discussion Paper Series 1401.

Heid, B., \& Larch, M. (2011). Migration, trade and unemployment, economics: The Open-Access, Open Assessment E-Journal 5, (2011-45).

Huang, W. (2008). Information lag and dynamic stability. Journal of Mathematical Economics, 44(2008), 513-529.

Jean, S., \& Jimenez, M. (2011). The unemployment impact of immigration in OECD countries. European Journal of Political Economy, 27(2), 241-256.

Kim, C. (2013). A condition for Hopf bifurcation to occur in equations of Lotka-Volterra type with delays. Differential Equations and Dynamical Systems, 21(3), 291-307.

Mircea, G., Neamţu, M., \& Opriş, D. (2011). Uncertain, stochastic and fractional dynamical systems with delay. Saarbrücken: LAP LAMBERT Academic Publishing.

Misra, A. K., \& Singh, A. K. (2011). A mathematical model for unemployment, IJTPC, pp. 2322-3138.

Misra, A. K., \& Singh, A. K. (2013). A delay mathematical model for the control of unemployment. Differential Equations and Dynamical Systems, 21(3), 291-307.

Nikolopoulos, C. V., \& Tzanetis, D. E. (2003). A model for housing allocation of a homeless population due to a natural disaster. Nonlinear Analysis, 4, 561-579.

OBR. (2013). Fiscal sustainability report. http://budgetresponsibility.independent.gov.uk/, Office for Budget Responsibility. July 2013.

Pissarides, C. A. (2011). Equilibrium in the labor market with search frictions. The American Economic Review, 101(4), 1092-1105.

Ruan, S. (1996). Bifurcation analysis of a chemostat model with a distributed delay. Journal of Mathematical analysis and applications, 204, 786-812.

Shimer, R. (2011). Reassessing the ins and outs of unemployment. Review of Economic Dynamics, 15(2), 127. 